\title{
ON SQUARE INTEGRABLE MARTINGALES
}

\author{
HIROSHI KUNITA AND SHINZO WATANABE
}

To Professor Kiyoshi Noshiro on the occasion of his 60th birthday.

\section{§ 0 . Introduction.}

Theory of real and time continuous martingales has been developed recently by P. Meyer $[8,9]$. Let $\left\{X_{t}, \mathfrak{\mho}_{t}\right\}$ be a square integrable martingale on a probability space $P$. He showed that there exists an increasing process $\langle X\rangle_{t}$ such that

$$
\left.\left.E\left(\left(X_{t}-X_{s}\right)^{2} / \mathfrak{F}_{s}\right)=E\left(\langle X\rangle_{t} / \mathfrak{F}_{s}\right)-\langle X\rangle_{s} \quad \text { a.e. } P \text { for } t\right\rangle s\right\rangle 0 \text {. }
$$

The above formula suggests us that some results concerning Brownian motion can be generalized to those of martingale. Actually, if $\langle X\rangle_{t} \equiv t, X_{t}$ is a Brownian motion (See [2, Theorem 11.9], also Theorem 2.3 of this paper), and in general, many continuous martingales may be obtained by time changes of Brownian motions ([3] and Theorem 3.1 of this paper).

Stochastic integral concerning martingale was defined by Meyer [9], Courrège [1] and in some special case, by Motoo and S. Watanabe [10]. In the present paper we shall discuss a formula on stochastic integral which is a generalization of Itô's formula [4] concerning Brownian motion. Let $X_{t}$ be a Brownian motion and $f$ be a $C^{2}$-class function. Itô's formula is the following:

$$
f\left(X_{t}\right)-f\left(X_{0}\right)=\int_{0}^{t} \frac{d f}{d x}\left(X_{s}\right) d X_{s}+\frac{1}{2} \int_{0}^{t} \frac{d^{2} f}{d x^{2}}\left(X_{s}\right) d s .
$$

We shall show in $\S 2$ that when $X_{t}$ is a continuous martingale, the above formula is still valid if $d s$ is replaced by $d\langle X\rangle_{s}$. When $X_{t}$ is not continuous, the formula becomes a more complicated form (See § 5). There, Lévy system introduced by one of the authors [11] plays an important role.

The formula on stochastic integral will be applied to two problems. In $\S 6$, we shall discuss the structure of multiplicative functionals of a Markov process. Roughly speaking, every multiplicative functional with mean 1 is factorized into two mutually orthogonal martingales; one is continuous multiplicative functional and the other is a jump type one. $\S 7$ is devoted to giving another proof of Lévy-Itô's decomposition of additive process (process with independent 
increments), using the formula on stochastic integral.

$\S 4$ is devoted to other problems. Let $\mathfrak{M}$ be the set of all square integrable martingales with mean 0 . When $\mathfrak{M}$ is defined on the probability space of a Hunt process, $\mathfrak{M}$ can be spanned by additive functionals. In particular, if $X_{t}$ is a martingale defined on the probability space of $N$-dimensional Brownian motion $\boldsymbol{B}=\left(B_{t}^{(1)}, \ldots \ldots, B_{t}^{N}\right)$, then $X_{t}$ is represented as $\sum_{i=1}^{n} \int_{0}^{t} \Phi_{s}^{(i)} d B_{s}^{(i)}$, where $\Phi_{s}^{(i)}$ are processes adapted to $\mathfrak{F}_{8}$.

\section{§1. Square integrable martingale.}

Let $(\Omega, \mathfrak{F}, P)$ be the basic probability space where $\mathfrak{F}$ is complete with respect to $P$, and suppose we are given an increasing family $\mathfrak{F}_{t}$ of $\sigma$-algebras $\mathfrak{F}_{t} \subset \mathfrak{F}$, $0 \leqq t<\infty$, each containing all $P$-null sets and $\mathfrak{F}_{t}=\underset{\varepsilon>0}{\cap} \mathfrak{F}_{t+\varepsilon}$. A non-negative random variable $T$ is called a stopping time if $\{T \leqq t\} \in \mathfrak{\mho}_{t}$ for every $t \geqq 0$. Given a stopping time $T$ we shall define

$$
\mathfrak{F}_{T}=\left\{A \in \mathfrak{F} ; \quad A \cap\{T \leqq t\} \in \mathfrak{F}_{t} \quad \text { for every } t \geqq 0\right\} .
$$

$\mathfrak{F}_{T}$ is clearly a $\sigma$-algebra containing all $P$-null sets such that if $T_{n} \downarrow T$ then $\bigcap_{n} \mathfrak{F}_{T_{n}}=\mathfrak{F}_{T^{1}}^{1}$.

Definition 1.1. $\mathfrak{F}_{t}$ is said to have no time of discontinuity if $T_{n} \uparrow T$ implies $\bigvee \mathfrak{F}_{T_{n}}=\mathfrak{F}_{T}$, where $\bigvee \mathfrak{F}_{T_{n}}$ denotes the smallest $\sigma$-algebra containing all $\mathfrak{F}_{T_{n}}$.

Definition 1.2.

(1.1) $\mathfrak{M}=\left\{\right.$ the set of all right continuous real valued processes $X_{t}$ adapted to the family $\mathfrak{F}_{t}$ such that $E\left(X_{t}^{2}\right)<\infty$ for every $t \in[0, \infty)$ and $E\left(X_{t} / \mathfrak{F}_{s}\right)=X_{s}$ for every $t \geqq s$. $\}$

(1.2) $\mathfrak{M}_{c}=\left\{X \in \mathfrak{M} ; X_{t}\right.$ is continuous in $t$ for almost all $\left.\omega\right\}$.

(1.3) $\mathfrak{M}^{l o c}=\left\{\right.$ the set of all processes $X_{t}$ such that there exists an increasing sequence $\left\{T_{n}\right\}$ of stopping times such as $T_{n} \uparrow \infty$ and $X_{t}^{(n)} \equiv X_{t \wedge T_{n}} \in \mathfrak{M}$ for every $n=1,2, \ldots \ldots$.$\} .$

(1.4) $\mathfrak{M}_{c}^{l o c}=\left\{X_{t} \in \mathfrak{M}^{l o c} ; \quad X_{t}\right.$ is continuous in $t$ for almost all $\left.\omega\right\}$. $X_{t} \in \mathfrak{M}$ is nothing but a square integrable martingale.

Definition 1.3.

(1.5) $\mathfrak{A}^{+}=\left\{\right.$the set of all natural increasing processes ${ }^{2)} A_{t}$ such that $E\left(A_{t}\right)<\infty$

1) $T, T_{n}, \ldots \ldots$ are stopping times.

2) For the definitions see [8] or Appendix. 
for every $t \in[0, \infty)\}$.

(1.6) $\mathfrak{X}^{+l o c}=\left\{\right.$ the set of all increasing processes $A_{t}$ such that there exists an increasing sequence $\left\{T_{n}\right\}$ such as $T_{n} \uparrow \infty$ and $A_{t}^{(n)} \equiv A_{t} \wedge T_{n} \in \mathfrak{A}^{+}$for every $\left.n.\right\}$

(1.7) $\mathfrak{U}=\left\{A_{t}=A_{t}^{(1)}-A_{t}^{(2)} ; A_{t}^{(i)} \in \mathfrak{A}^{+} i=1,2\right\}$.

$\mathfrak{A}^{l o c}$ is defined in a similar way. Finally $\mathfrak{A}_{c}^{+}, \mathfrak{A}_{c}^{+l o c}, \ldots \ldots$ are all continuous elements in $\mathfrak{A}^{+}, \mathfrak{A}^{+l o c}, \ldots . .$. respectively.

Theorem 1.1. (i) For $X, Y \in \mathfrak{M}$ there exists a unique (up to equivalence) $<X, Y>\in \mathfrak{A}$ such that

(1.8) $E\left[\left(X_{t}-X_{s}\right)\left(Y_{t}-Y_{s}\right) / \mathfrak{F}_{s}\right]=E\left[\langle X, Y\rangle_{t}-\langle X, Y\rangle_{s} / \mathfrak{F}_{s}\right]$ a.e. for every $t \geqq s \in[0, \infty)$.

(ii) For $X, Y \in \mathfrak{M}^{l o c}$ there exists a unique (up to equivalence) $\langle X, Y\rangle \in \mathfrak{H}^{l o c}$ such that

(1.9) $E\left[\left(X_{t \wedge T_{n}}-X_{s \wedge T_{n}}\right)\left(Y_{t \wedge T_{n}}-Y_{s \wedge T_{n}}\right) / \mathfrak{F}_{s}\right]=E\left[\langle X, Y\rangle_{t \wedge T_{n}}-\langle X, Y\rangle_{s} \wedge T_{n} / \mathfrak{F}_{s}\right]$ a.e. for every $t \geqq s \in[0, \infty)$ and every $T_{n}$ such that $X_{t \wedge T_{n}}, Y_{t \wedge T_{n}} \in \mathfrak{M}$.

Proof. If $X=Y \in \mathfrak{M}$, the existence and the uniqueness of $\langle X, Y\rangle$ was proved in Meyer ([8, 9; Th. 4, Th. 5]). For $X, Y \in \mathfrak{M}$ set $\langle X, Y\rangle=1 / 4(\langle X+Y$, $X+Y\rangle-\langle X-Y, X-Y\rangle)$. Then (1.8) holds and the uniqueness is easy to see. If $X, Y \in \mathfrak{M}^{l o c}$, take $T_{n} \uparrow \infty$ such that $X_{t}^{(n)}=X_{t \wedge T_{n}}, Y_{t}^{(n)}=Y_{t \wedge T_{n}} \in \mathfrak{M}$ for every $n$. Then $\left\langle X^{(n)}, Y^{(n)}\right\rangle \in \mathfrak{A}$ for every $n$ and by the uniqueness of $\left\langle X^{(n)}, Y^{(n)}\right\rangle$, we have

$$
\left\langle X^{(m)}, Y^{(m)}\right\rangle_{t \wedge T_{n}}=\left\langle X^{(n)}, Y^{(n)}\right\rangle_{t}, \quad m \geqq n \quad t \in[0, \infty) .
$$

Hence there exists a unique $\langle X, Y\rangle \in \mathfrak{A}^{\text {loc }}$ such that

$$
\left\langle X^{(n)}, Y^{(n)}\right\rangle_{t}=\langle X, Y\rangle_{t \wedge T_{n}}
$$

and (1.9) is clearly satisfied.

Theorem 1.2. $\langle X, Y\rangle$ defined in the previous theorem is continuous if both $X$ and $Y$ are quasi-left continuous $\left(X_{t}\right.$ is called quasi-left continuous if for every increasing sequence of stopping times $T_{n} \uparrow T, X_{T_{n}} \rightarrow X_{T}$ a.s. on $\left.\{T<\infty\}\right):$ in particular if one of the following conditions is satisfied;

(i) Both $X$ and $Y$ are continuous,

(ii) $\left\{\mathfrak{\mho}_{t}\right\}$ has no time of discontinuity.

Proof. Take any stopping time $S$ such that $X_{t \wedge s}$ and $Y_{t \wedge S}$ are uniformly integrable. Then quasi-left continuity implies that $X_{t \wedge s}$ and $Y_{t} \wedge s$ are regular and so $\langle X, Y\rangle$ is continuous by Th. 3 of [8]. Clearly (1) implies the quasi-left 
continuity of $X$ and $Y$. If (2) is satisfied then $X_{t \wedge T_{n}}=E\left(X_{t} / \mathfrak{F}_{t} \wedge T_{n}\right) \rightarrow E\left(X_{t} / \mathfrak{F}_{t} \wedge T\right)$ $=X_{t} \wedge$ r

For simplicity, we shall write $\langle X, X\rangle$ as $\langle X\rangle$.

Definition 1.4. A sequence $\left\{T_{n}\right\}$ of stopping times is called a chain if

$$
T_{0}=0, T_{n} \leqq T_{n+1} \text { and } \lim _{n \rightarrow \infty} T_{n}=+\infty \text { a.e. }
$$

Let $X_{t}$ be a right-continuous process having left limits in $[0, \infty)$ adapted to the family $\mathfrak{F}_{t}$. A chain $\left\{T_{n}\right\}$ is called an $\left(\varepsilon, X_{t}\right)$-chain or $\varepsilon$-chain for $X_{t}$ if, for almost all $\omega$,

$$
\sup _{t, s \in\left[T_{n}, T_{n+1}\right)}\left|X_{t}-X_{s}\right|<\varepsilon .
$$

For every right continuous process $X_{t}$ having left limits, an $\left(\varepsilon, X_{t}\right)$-chain always exists.

Let $C=\left\{T_{n}\right\}$ and $C^{\prime}=\left\{T_{n}^{\prime}\right\}$ be two chains. $C^{\prime}$ is called a subdivision of $C\left(C^{\prime} \prec C\right)$ if $\left\{T_{n}(\omega)\right\} \subset\left\{T_{n}^{\prime}(\omega)\right\}$ almost all $\omega$. For given $C$ and $C^{\prime}$ there exists $C^{\prime \prime}$ such that $C^{\prime \prime} \prec C$ and $C^{\prime \prime} \prec C^{\prime}$.

Theorem 1. 3. If $X \in \mathfrak{M}_{c}^{20 c}$, then there exists a sequence $C_{k}=\left\{T_{n}^{(k)}\right\}$ of chains such that $C_{k}>C_{k+1}, \sup _{n}\left|T_{n+1}^{(k)}-T_{n}^{(k)}\right| \rightarrow 0(k \rightarrow \infty)$ and for almost all $\omega$

$$
\sum_{n}\left(X_{T_{n}^{(k)} \wedge_{t}}-X_{T_{n-1}^{(k)} \wedge_{t}}\right)^{2} \rightarrow\langle X\rangle_{t}-\langle X\rangle_{0} \quad(k \rightarrow \infty) \quad \text { for every } t \geqq 0 .
$$

Proof. Let $C_{k}=\left\{T_{n}^{(k)}\right\}, k=1,2, \ldots \ldots$ be a sequence of chains such that $C_{k}>C_{k+1}$ and $C_{k}$ is a $1 / 2^{k}$-chain for $X_{t},\langle X\rangle_{t}$ and $A_{t} \equiv t$.

Clearly we may assume that $X \in \mathfrak{M}_{c}$. Then writing $T_{n \wedge}^{(k)} \wedge t=\sigma_{n}$ for simplicity,

$$
\begin{aligned}
& E\left[\left\{\sum_{n=1}^{\infty}\left(X_{\sigma_{n}}-X_{\sigma_{n-1}}\right)^{2}-\left\langle\langle X\rangle_{t}-\langle X\rangle_{0}\right)\right\}^{2}\right] \\
= & E\left[\left\{\sum_{n=1}^{\infty}\left(\left(X_{\sigma_{n}}-X_{\sigma_{n-1}}\right)^{2}-\left\langle\langle X\rangle_{\sigma_{n}}-\langle X\rangle_{\sigma_{n-1}}\right)\right)\right\}^{2}\right] \\
= & E\left(\sum _ { n } \sum _ { m } \{ ( X _ { \sigma _ { n } } - X _ { \sigma _ { n - 1 } } ) ^ { 2 } - ( \langle X \rangle _ { \sigma _ { n } } - \langle X \rangle _ { \sigma _ { n - 1 } } ) \} \left\{\left(X_{\sigma_{m}}-X_{\sigma_{m-1}}\right)^{2}\right.\right. \\
& \left.\left.-\left(\langle X\rangle_{\sigma_{m}}-\langle X\rangle_{\sigma_{m-1}}\right)\right\}\right]
\end{aligned}
$$

and noting

$$
\begin{array}{r}
E\left[\left(X_{\sigma_{n}}-X_{\sigma_{n-1}}\right)^{2}-\left(\langle X\rangle_{\sigma_{n}}-\langle X\rangle_{\sigma_{n-1}}\right) / \mho_{\sigma_{n-1}}\right]=0 \\
=E\left[\sum_{n}\left\{\left(X_{\sigma_{n}}-X_{\sigma_{n-1}}\right)^{2}-\left(\langle X\rangle_{\sigma_{n}}-\langle X\rangle_{\sigma_{n-1}}\right)\right\}^{2}\right]
\end{array}
$$




$$
\begin{aligned}
& \leqq 2 E\left[\sum_{n}\left\{\left(X_{\sigma_{n}}-X_{\sigma_{n-1}}\right)^{4}+\left(\langle X\rangle_{\sigma_{n}}-\langle X\rangle_{\sigma_{n-1}}\right)^{2}\right\}\right] \\
& \leqq \frac{2}{2^{k}} E\left[\Sigma\left(X_{\sigma_{n}}-X_{\sigma_{n-1}}\right)^{2}\right]+\frac{2}{2^{k}} E\left[\sum_{n}\left(\langle X\rangle_{\sigma_{n}}-\langle X\rangle_{\sigma_{n-1}}\right)\right] \\
& \leqq \frac{1}{2^{k-2}} E\left[\left(X_{t}-X_{0}\right)^{2}\right] .
\end{aligned}
$$

Hence (1.11) holds.

Corollary. If $\mathfrak{M}_{c}^{l o c} \cap \mathfrak{A}_{c}^{l o c} \ni X$ then $X_{t}-X_{0} \equiv 0$.

Proof. If $X \in \mathfrak{M}_{c} \cap \mathfrak{A}_{c}, \quad X_{t}$ is written as $X_{t}=A_{t}-B_{t}$ where $A, B \in \mathfrak{A}_{c}^{+}$. Then, if $\left\{T_{n}\right\}$ is an $(\varepsilon, X)$-chain,

$$
\sum_{n=1}^{\infty}\left(X_{T_{n} \wedge t}-X_{T_{n-1} \wedge_{t}}\right)^{2} \leqq \varepsilon \sum_{n=1}^{\infty}\left|X_{T_{n} \wedge t}-X_{T_{n-1} \wedge t}\right| \leqq \varepsilon\left(A_{t}+B_{t}\right) \rightarrow 0 \quad(\varepsilon \downarrow 0) .
$$

By Theorem 1.3, $\langle X\rangle_{t}-\langle X\rangle_{0}=0$ which concludes that $X_{t}=X_{0}$ a.e.

Remark. This corollary is a particular case of a general uniqueness theorem of Meyer [9].

\section{$\S 2$. Formula on stochastic integral I.}

We shall first define the stochastic integral of the form $Y_{t}=\int_{0}^{t} \Phi_{s} d X_{s}$, where $X_{t}$ is a martingale and $\Phi_{t}$ is a process adapted to the family $\mathfrak{F}_{t}, t>0$ and satisfying conditions described below. Set

(2.1) $\Phi=\left\{\Phi_{t}(\omega) ;(t, \omega)\right.$-measurable real valued process such that $\Phi_{T}(\omega)$ is $\mathfrak{F}_{T^{-}}$ measurable for each stopping time $T\}$,

(2.2) $\Phi_{r c}=\left\{\Phi_{t}(\omega)\right.$; bounded right continuous process having left-hand limits $\}$, and define for $\varphi \in \mathfrak{A}^{+}$semi norms \|\|$_{\varphi}(t)$ over $\Phi$ by $\|\Phi\|_{\varphi}(t)=E\left(\int_{0}^{t} \Phi_{s}^{2} d \varphi_{s}\right)^{\frac{1}{2}}$.

Set $L_{2}(\varphi)=\Phi \cap \bar{\Phi}_{r c}$, where $\bar{\Phi}_{r c}$ is the closure of $\Phi_{r c}$ with respect to seminorms .. $\|_{\varphi}(t)$.

Theorem 2.1. For every $X \in \mathfrak{M}$ and $\Phi \in L_{2}(\langle X\rangle)$, there exists a unique $Y \in \mathfrak{M}$ satisfying

$$
\langle Y, Z\rangle_{t}=\int_{0}^{t} \Phi_{s} d\langle X, Z\rangle_{s} \quad \text { a.e. } P \text { for any } Z \in \mathfrak{M} \text {. }
$$

Definition 2. 1.3) $Y$ of the above theorem is called the stochatic integral of $\Phi$ by $X$ and is denoted by $Y_{t}=\int_{0}^{t} \Phi_{s} d X_{s}$.

Before the proof, we prepare the following

Lemma 2.1. Let $X, Y \in \mathfrak{M}$ and $\Phi^{\prime}, \Phi^{\prime \prime} \in \Phi$. Suppose that

3) This definition of stochastic integral is due to Motoo, Watanabe [10]. 


$$
\begin{gathered}
\left\|\Phi^{\prime}\right\|_{<X>}(t)<\infty \text { and }\left\|\Phi^{\prime \prime}\right\|_{<Y>}(t)<\infty \\
\text { Then } \int_{0}^{t} \Phi_{s}^{\prime} \Phi_{s}^{\prime \prime} d\langle X, Y\rangle_{s} \in \mathfrak{U}
\end{gathered}
$$

and

$$
E\left(\left|\int_{0}^{t} \Phi_{s}^{\prime} \Phi_{s}^{\prime \prime} d\langle X, Y\rangle_{s}\right|\right) \leqq\left\|\Phi^{\prime}\right\|<X>(t)\left\|\Phi^{\prime \prime}\right\|<Y>(t) .
$$

Proof. Since

$$
\Delta\langle a X+b Y\rangle_{t}=a^{2} \Delta\langle X\rangle_{t}+2 a b \Delta\langle X, Y\rangle_{t}+b^{2} \Delta\langle Y\rangle_{t} \geqq 0,{ }^{4)}
$$

we have

$$
\left|\Delta\langle X, Y\rangle_{t}\right| \leqq\left(\Delta\langle X\rangle_{t}\right)^{\frac{1}{2}}\left(\Delta\langle Y\rangle_{t}\right)^{\frac{1}{2}} \leqq \frac{1}{2}\left(\Delta\langle X\rangle_{t}+\Delta\langle Y\rangle_{t}\right) \quad \text { a.e. } P \text {. }
$$

Set $\varphi_{t}=\langle X\rangle_{t}+\langle Y\rangle_{t}$, then there exist $\Psi^{1}, \Psi^{2}, \Psi^{3} \in \Phi$ such that

$$
\langle X\rangle_{t}=\int_{0}^{t} \Psi_{s}^{1} d \varphi_{s},\langle X, Y\rangle_{t}=\int_{0}^{t} \Psi_{s}^{2} d \varphi_{s},\langle Y\rangle_{t}=\int_{0}^{t} \Psi_{s}^{3} d \varphi_{s} .
$$

In fact we only have to set for example

$$
\Psi_{t}^{1}=\varlimsup_{h \downarrow 0} \frac{\langle X\rangle_{t}-\langle X\rangle_{t-h}}{\varphi_{t}-\varphi_{t-h}}
$$

Now since

$$
\langle a X+b Y\rangle_{t}=\int_{0}^{t}\left(a^{2} \Psi_{s}^{1}+2 a b \Psi_{s}^{2}+b^{2} \Psi_{s}^{3}\right) d \varphi_{s},
$$

there exists $\widetilde{\Omega}_{a b} \in \mathfrak{F}$ with $P\left(\widetilde{\Omega}_{a b}\right)=1$ such that if $\omega \in \widetilde{\Omega}_{a b}$ there exists $T_{a b}(\omega) \subset[0, \infty)$ with $\int_{[0, t] \cap T_{a b}(\omega)} d \varphi_{s}(\omega)=0$ and for $s \notin T_{a b}(\omega)$,

$$
a^{2} \Psi_{s}^{1}(\omega)+2 a b \Psi_{s}^{2}(\omega)+b^{2} \Psi_{s}^{3}(\omega) \geqq 0 .
$$

Set $\widetilde{\Omega}=\bigcap_{a, b \text { rational }} \widetilde{\Omega}_{a b}$ and $T(\omega)=\cup T_{a b}(\omega)$ for $\omega \in \widetilde{\Omega}$. Then $P(\widetilde{\Omega})=1$ and if $\omega \in \widetilde{\Omega}$ then $\int_{[0, t] \cap T} d \varphi_{s}=0$ for every $t$ and if $s \notin T(\omega)$

$$
a^{2} \Psi_{s}^{1}(\omega)+2 a b \Psi_{s}^{2}(\omega)+b^{2} \Psi_{s}^{3}(\omega) \geqq 0
$$

for every rational $a, b$ and hence for every real $a, b$. Therefore, for every real $a, b$ and $s \notin T(\omega)$

$$
a^{2} \Phi_{s}^{\prime}(\omega)^{2} \Psi_{s}^{1}(\omega)+2 a b \Phi_{s}^{\prime}(\omega) \Phi_{s}^{\prime \prime}(\omega) \Psi_{s}^{2}(\omega)+b^{2} \Phi_{s}^{\prime \prime}(\omega)^{2} \Psi_{s}^{3}(\omega) \geqq 0
$$

and so integrating by $d \varphi_{s}$ on $\left[t_{1}, t_{2}\right]$

4) $\Delta\langle X\rangle_{t}=\langle X\rangle_{t+h}-\langle X\rangle_{t}$. 


$$
\begin{aligned}
a^{2} \int_{t_{1}}^{t_{2}} \Phi_{s}^{\prime}(\omega)^{2} d\langle X\rangle_{s}(\omega)+2 a b \int_{t_{1}}^{t_{2}} \Phi_{s}^{\prime}(\omega) & \Phi_{s}^{\prime \prime}(\omega) d\langle X, Y\rangle_{s}(\omega) \\
& +b^{2} \int_{t_{1}}^{t_{2}} \Phi_{s}^{\prime \prime}(\omega)^{2} d\langle Y\rangle_{s}(\omega) \geqq 0, \quad \omega \in \widetilde{\Omega} .
\end{aligned}
$$

Putting $t_{1}=0$ and $t_{2}=t$ and taking the expectation, we get

$$
a^{2}\left\|\Phi^{\prime}\right\|<X>(t)^{2}+2 a b E\left(\int_{0}^{t} \Phi_{s}^{\prime} \Phi_{s}^{\prime \prime} d\langle X, Y\rangle_{s}\right)+b^{2}\left\|\Phi^{\prime \prime}\right\|<X>(t) \geqq 0 .
$$

Thus we get (2.4). From (2.5), we have also that if $\omega \in \widetilde{\Omega}$,

$$
\Delta_{t} \int \Phi_{s}^{\prime}(\omega) \Phi_{s}^{\prime \prime}(\omega) d\langle X, Y\rangle_{s}(\omega) \leqq-1 .\left\{\Delta_{t} \int \Phi_{s}^{\prime}(\omega)^{2} d\langle X\rangle_{s}(\omega)+\Delta_{t} \int \Phi_{s}^{\prime \prime}(\omega)^{2} d\langle Y\rangle_{s}(\omega)\right\}
$$

and this implies $\int_{0}^{t} \Phi_{s}^{\prime} \Phi_{s}^{\prime \prime} d<X, Y>_{s} \in \mathfrak{A}$.

Proof of Theorem 2.1. First we consider the case that $\Phi$ is a step function, i.e., there is an increasing sequence of stopping times $\left\{T_{n}\right\}$ such that $T_{n} \rightarrow \infty$ and $\Phi_{s}(\omega)=\Phi_{T_{n-1}}(\omega)$ if $T_{n-1} \leqq s<T_{n}$.

Define

$$
Y_{t}=\sum_{n} \Phi_{T_{n-1} \wedge t}\left(X_{T_{n} \wedge t}-X_{T_{n-1} \wedge t}\right)
$$

It is easy to see that $Y_{t}$ belongs to $\mathfrak{M}$ and satisfies (2.3). Now, let $\Phi$ be any element of $L_{2}\left(\langle X>)\right.$. We can choose a sequence of step functions $\left\{\Phi^{n}\right\} \subset \Phi$ such that $\left\|\Phi-\Phi^{n}\right\|_{<X>}(t) \rightarrow 0$. Set $Y^{n}=\int \Phi^{n} d X$. Then, by (2.3) we get

$$
E\left(\left|Y_{t}^{n}-Y_{t}^{m}\right|^{2}\right)=E\left(\left\langle Y^{n}-Y^{m}\right\rangle_{t}\right)=E\left(\int_{0}^{t}\left(\Phi_{s}^{n}-\Phi_{s}^{m}\right)^{2} d\langle X\rangle_{s}\right) \rightarrow 0,
$$

so that there exists $Y_{t}=\lim _{n \rightarrow \infty} Y_{t}^{n} \in \mathfrak{M}$. From Lemma 2.1, we have

$$
\begin{aligned}
& E\left(\left|\left\langle Y-Y^{n}, Z\right\rangle_{t}\right|\right) \leqq E\left(\left|\left\langle Y-Y^{n}\right\rangle_{t}\right|\right)^{1 / 2} E\left(\langle Z\rangle_{t}\right)^{1 / 2} \underset{n \rightarrow \infty}{\rightarrow} 0, \\
& E\left(\left|\int_{0}^{t}\left(\Phi_{s}-\Phi_{s}^{n}\right) d\langle X, Z\rangle_{s}\right|\right) \leqq\left\|\Phi-\Phi^{n}\right\|_{\langle X\rangle}(t)^{1 / 2} E\left(\langle Z\rangle_{t}\right)^{1 / 2} \rightarrow 0 .
\end{aligned}
$$

Therefore

$$
\langle Y, Z\rangle_{t}=\lim _{n \rightarrow \infty}\left\langle Y^{n}, Z\right\rangle_{t}=\lim _{n \rightarrow \infty} \int_{0}^{t} \Phi_{s}^{n} d\langle X, Z\rangle=\int_{0}^{t} \Phi_{s} d\langle X, Z\rangle_{s} .
$$

The following formula is a generalization of a formula of K. Itô [4] in the case $X_{t}$ is a Brownian motion and $\varphi_{t}=0$.

Theorem 2.2. Let $F \in C^{2}\left(R^{N}\right)=$ the set of all twice continuously differentiable 
functions on $R^{N}$. Let $X_{t}^{(1)}, \ldots \ldots, X_{t}^{(N)} \in \mathfrak{M}_{c}^{l o c}$ and $\varphi_{t}^{(1)}, \ldots \ldots, \varphi_{t}^{(n)} \in \mathfrak{A}_{c}^{l o c}$. Then, setting $A_{t}^{(i)}=X_{t}^{(i)}+\varphi_{t}^{(i)}$ and $\boldsymbol{A}_{t}=\left(A_{t}^{(1)}, \ldots \ldots, A_{t}^{(n)}\right)$, we have

$$
\begin{aligned}
F\left(\boldsymbol{A}_{t}\right)-F\left(\boldsymbol{A}_{0}\right)= & \sum_{i=1}^{N} \int_{0}^{t} \frac{\partial F}{\partial x^{2}}\left(\boldsymbol{A}_{s}\right) d X_{s}^{(i)}+\sum_{i, j=1}^{N} \frac{1}{2} \int_{0}^{t} \frac{\partial^{2} F}{\partial x^{i} \partial x^{j}}\left(\boldsymbol{A}_{s}\right) d\left\langle X^{(i)}, X^{(j)}\right\rangle_{s} \\
& +\sum_{i=1}^{N} \int_{0}^{t} \frac{\partial F}{\partial x^{i}}\left(\boldsymbol{A}_{s}\right) d \varphi_{s}^{(i)} .
\end{aligned}
$$

Proof. For simplicity, we assume that $N=1$. Let $X \in \mathfrak{M}_{c}^{l o c}$ and $\varphi \in \mathfrak{A}_{c}^{l o c}$. Set for $M>0$,

$$
T_{M}=\left\{\begin{array}{l}
\text { inf }\left\{t ;\left|X_{t}\right|>M / 2 \text { or }\left|\varphi_{t}\right|>M / 2\right\} \\
\infty \text { if }\{\}=\phi .
\end{array}\right.
$$

If we can prove (2.7) for $X_{t}^{M}=X_{t \wedge T_{M}}$ and $\varphi_{t}^{M}=\varphi_{t \wedge T_{M}}$, then letting $M \rightarrow \infty$, we obtain (2.7). So without loss of generality we can assume that $\left|X_{t}\right|$ and $\left|\varphi_{t}\right|$ are bounded $(\leq M / 2)$. Let $F \in C^{2}\left(R^{1}\right)$. For $k=1,2, \ldots \ldots$, we take a sequence of chains $C_{k}=\left\{T_{n}^{(k)}\right\}$ of stopping times such that $C_{k}>C_{k+1}$ and $C_{k}$ is a $1 / 2^{k}$-chain for $X_{t},\langle X\rangle_{t},|\varphi|_{t}$ and $t$, here $|\varphi|_{t}$ is the total variation of $\varphi_{t}$. Writing $T_{n}^{(k)} \wedge t=\sigma_{n}$, we have

$$
\begin{aligned}
F\left(A_{t}\right)-F\left(A_{0}\right) & =\sum_{n=1}^{\infty}\left\{F\left(A_{\sigma_{n}}\right)-F\left(A_{\sigma_{n-1}}\right)\right\} \\
& =\sum_{n=1}^{\infty} F^{\prime}\left(A_{\sigma_{n-1}}\right)\left(A_{\sigma_{n}}-A_{\sigma_{n-1}}\right)+\frac{1}{2} \sum_{n=1}^{\infty} F^{\prime \prime}\left(\xi_{n-1}\right)\left(A_{\sigma_{n}}-A_{\sigma_{n-1}}\right)^{2} \\
& =I_{1}+I_{2},
\end{aligned}
$$

where $A_{\sigma_{n-1}} \leqq \xi_{n-1} \leqq A_{\sigma_{n}}$ or $A_{\sigma_{n-1}} \leqq \xi_{n-1} \leqq A_{\sigma_{n}}$. By the definition of stochastic integral it is easy to see that

$$
I_{1} \rightarrow \int_{0}^{t} F^{\prime}\left(A_{s}\right) d X_{s}+\int_{0}^{t} F^{\prime}\left(A_{s}\right) d \varphi_{s}
$$

when $k \rightarrow \infty$. Set

$$
\begin{aligned}
I_{2}^{\prime} & =\frac{1}{2} \sum_{n=1}^{\infty} F^{\prime \prime}\left(A_{\sigma_{n-1}}\right)\left(A_{\sigma_{n}}-A_{\sigma_{n-1}}\right)^{2} \\
& =\frac{1}{2} \sum_{n=1}^{\infty} F^{\prime \prime}\left(A_{\sigma_{n-1}}\right)\left(X_{\sigma_{n}}-X_{\sigma_{n-1}}\right)^{2} \\
& +\sum_{n=1}^{\infty} F^{\prime \prime}\left(A_{\sigma_{n-1}}\right)\left(X_{\sigma_{n}}-X_{\sigma_{n-1}}\right)\left(\varphi_{\sigma_{n}}-\varphi_{\sigma_{n-1}}\right) \\
& +-\frac{1}{2} \sum_{n=1}^{\infty} F^{\prime \prime}\left(A_{\sigma_{n-1}}\right)\left(\varphi_{\sigma_{n}}-\varphi_{\sigma_{n-1}}\right)^{2}
\end{aligned}
$$




$$
=I_{3}+I_{4}+I_{5}
$$

Then

$$
\left|I_{4}\right| \leqq \sup _{x \in[-M, M]}\left|F^{\prime \prime}(x)\right| \frac{1}{2^{k}} \sum_{n=1}^{\infty}\left|\varphi_{\sigma_{n}}-\varphi_{\sigma_{n-1}}\right| \leqq\left. 2_{2^{k}}^{1} \sup _{x \in[-M, M]}\left\|F^{\prime \prime}(x)\right\| \varphi\right|_{t} \rightarrow 0(k \rightarrow \infty) .
$$

In the same way, $\left|I_{5}\right| \rightarrow 0$ as $k \rightarrow \infty$. Now, by the same calculation as in (1.12),

$$
\begin{aligned}
& E\left[\left\{I_{3}-1-\sum_{n=1}^{\infty} F^{\prime \prime}\left(A_{\sigma_{n-1}}\right)\left(\langle X\rangle_{\sigma_{n}}-\langle X\rangle_{\sigma_{n-1}}\right)\right\}^{2}\right] \\
\leq & E\left[\sum_{n=1} F^{\prime \prime}\left(A_{\sigma_{n-1}}\right)^{2}\left(X_{\sigma_{n}}-X_{\sigma_{n-1}}\right)^{4}+\sum_{n=1}^{\infty} F^{\prime \prime}\left(A_{\sigma_{n-1}}\right)^{2}\left(\langle X\rangle_{\sigma_{n}}-\langle X\rangle_{\sigma_{n-1}}\right)^{2}\right] \\
\leq & \sup _{x \in[-M, M]}\left|F^{\prime \prime}(x)\right|\left\{\frac{1}{2^{2 k}} E\left(\left(X_{t}-X_{0}\right)^{2}\right)+\frac{1}{2^{k}} E\left(\langle X\rangle_{t}-\langle X\rangle_{0}\right)\right\} \rightarrow 0
\end{aligned}
$$

as $k \rightarrow \infty$, Also

$$
\begin{aligned}
& E\left[\left|\frac{1}{2} \int_{0}^{t} F^{\prime \prime}\left(A_{s}\right) d\langle X\rangle_{s}-\frac{1}{2} \sum_{n=1}^{\infty} F^{\prime \prime}\left(A_{\sigma_{n-1}}\right)\left(\langle X\rangle_{\sigma_{n}}-\langle X\rangle_{\sigma_{n-1}}\right)\right|\right] \\
& \leqq-\frac{1}{2} \delta(k) E\left(\langle X\rangle_{t}-\langle X\rangle_{0}\right) \rightarrow 0 \quad(k \rightarrow \infty)
\end{aligned}
$$

where $\delta(k)=\sup _{\substack{x, y \in[-M, M] \\|x-y|<1 / 2^{k-1}}}|F(x)-F(y)|$. Hence $I_{2}^{\prime} \rightarrow \frac{1}{2} \int_{0}^{t} F^{\prime \prime}\left(A_{s}\right) d\langle X\rangle_{s}$. It is easy to see that $E\left|I_{2}-I_{2}^{\prime}\right| \rightarrow 0$. Therefore $I_{2} \rightarrow \frac{1}{2} \int_{0}^{t} F^{\prime \prime}\left(A_{s}\right) d\langle X\rangle_{s}$ and the proof is complete.

As an application of this formula we shall prove a theorem of P. Lévy (Theorem 11.9, Chap. VII of Doob [2]).

Theorem 2.3. Let $X_{t}^{(i)} \in \mathfrak{M}_{c}^{l o c}, \quad i=1,2, \ldots \ldots, N$ and $\left\langle X^{(i)}, X^{(j)}\right\rangle_{t}=\delta_{i j} t$, then $X_{t}=\left(X_{t}^{(1)}, \ldots \ldots, X_{t}^{(N)}\right)$ is an $N$-dimensional Brownian motion, that is,

(i) $X_{t}-X_{s}$ is independent of $\mathfrak{F}_{s}(t \geqq s)$,

(ii) $X_{t}-X_{s}$ is Gaussian i.e. $E\left(e^{i<\xi, X_{t}-X_{s}>}\right)=e^{-\frac{1}{2}|\xi|^{2}(t-s)}$.

Proof. For $\xi, x \in R^{N}$, let $F(x)=e^{i<\xi, x>}$ then from (2.7) we have

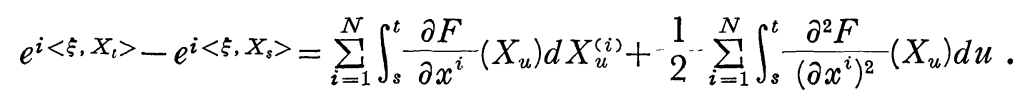

Take any $A \in \mathfrak{F}_{s}$. Then noting $E\left(\int_{s}^{t} \frac{\partial F}{\partial x^{i}}\left(X_{u}\right) d X_{u} / \mathfrak{F}_{s}\right)=0$, we have

$$
E\left(e^{i<\xi, X_{l}>} ; A\right)-E\left(e^{i<\xi, X_{s}>} ; A\right)=-\frac{1}{2} \sum_{i=1}^{N}\left(\xi^{i}\right)^{2} E\left(\int_{s}^{t} e^{i<\xi, X_{u}>} d u ; A\right) .
$$


Or equivalently,

$$
E\left(e^{i<\xi, X_{t}-X_{s}>} ; A\right)=P(A)-\frac{|\xi|^{2}}{2} \int_{s}^{t} E\left(e^{i<\xi, X_{u}-X_{s}>} ; A\right) d u
$$

From this integral equation we see at once

$$
E\left(e^{i<\xi, X_{t}-X_{s}>} ; A\right)=P(A) e^{-\frac{1}{2}|\xi|^{2}(t-s)}
$$

Since this holds for every $A \in \mathfrak{F}_{s}$, we get

$$
E\left(e^{i<\xi, X_{t}-X_{s}>} / \mathfrak{F}_{s}\right)=e^{-\frac{1}{2}|\xi|^{2}(t-s)}
$$

and (i) and (ii) follows from this.

\section{$\S 3$. Time change (optional sampling) of an $\mathrm{N}$-dimensional Brownian motion.}

As before we assume that we are given $(\Omega, \mathfrak{F}, P)$ and an increasing and right continuous family $\mathfrak{F}_{t}$.

Definition 3.1. $\tau=\left\{T_{u}, u \in[0, \infty)\right\}$ is called a time change function with respect to $\mathfrak{F}$, if

(i) for each $u \in[0, \infty), T_{u}$ is a stopping time and $T_{u}<\infty$.

(ii) for almost all $\omega, u \in[0, \infty) \rightarrow T_{u} \in[0, \infty)$ is a continuous and increasing function.

Definition 3.2. For a right continuous process $X$ adapted to the family $\mathfrak{F}_{t}$ and a time change function $\tau=\left\{T_{u}\right\}$, we define a new process $\left\{\tilde{X}_{u}, \tilde{\widetilde{F}}_{u}\right\}$ by $\tilde{X}_{u}=X_{T_{u}}$ and $\tilde{\mathfrak{F}}=\mathfrak{F}_{T_{u}}$. We shall call $\left\{\tilde{X}_{u}, \tilde{\mathfrak{F}}_{u}\right\}$ is obtained from $\left\{X_{t}, \mathfrak{F}_{t}\right\}$ by time change with respect to $\tau$.

By a fundamental theorem (optional sampling theorem) due to Doob [2], $X_{t} \in \mathfrak{M}_{c}^{l o c}\left(\mathfrak{F}_{t}\right)^{5)}$ implies $\tilde{X}_{u} \in \mathfrak{M}^{l o c}\left(\tilde{F}_{u}\right)$. Dubins, Schwarz [3] proved that a large class of 1-dimensional continuous martingales can be obtained from 1-dimensional Brownian motion by time change. We shall generalize this theorem in the following manner.

Theorem 3.1. Let $X_{t}=\left(X_{t}^{(1)}, \ldots \ldots, X_{t}^{(N)}\right)\left(X_{0}=x \in R^{N}\right)$ be a continuous process with values in $R^{N}$ adapted to the family $\mathfrak{F}_{t}$ such that for every harmonic function $h(x)$ on $R^{N}$

$$
X_{t}^{h}=h\left(X_{t}\right)-h\left(X_{0}\right) \in \mathfrak{M i}_{c}^{l o c}
$$

5) $\mathfrak{M}\left(\mathfrak{\mho}_{t}\right)$ is the space of square integrable martingales relative to $\left(\mathfrak{F}_{t}, P\right)$. 
and for every open interval $I \subset[0, \infty)$

$$
P\left(\sup _{t, s \in I}\left|X_{t}-X_{s}\right|>0\right)=1 .
$$

Then there exists an $N$-dimensional Brownian motion process $\left\{B_{u}, \mathfrak{F}_{u}^{*}\right\}$ and $X_{t}$ is obtained from it by time change.

Remark. If $N=1$, then every harmonic function is of the form $a x+b$ and so this theorem contains the result of [3].

Proof. Since $h(x)=x^{i}, \quad x=\left(x^{1}, \ldots \ldots, x^{N}\right)$ is harmonic, $X_{t}^{(i)}-X_{0}^{(i)} \in \mathfrak{M}_{c}^{l o c}$, $i=1,2, \ldots \ldots, N$. We shall first prove that there exists $A \in \mathfrak{A}_{c}^{l o c}$ such that $\left\langle X^{(i)}, X^{(j)}\right\rangle_{t}=\delta_{i, j} A_{t}, \quad i, j=1, \ldots \ldots, N$. Since $h(x)=x^{i} x^{j} \quad(i \neq j)$ is harmonic

$$
X_{t}^{(i)} X_{t}^{(j)}-X_{0}^{(i)} X_{0}^{(j)} \in \mathfrak{M}_{c}^{l o c} .
$$

By Theorem 2.2,

$$
X_{t}^{(i)} X_{t}^{(j)}-X_{0}^{(i)} X_{0}^{(j)}-\sum_{k=1}^{N} \int_{0}^{t} \frac{\partial h}{\partial x^{k}}\left(X_{s}\right) d X_{s}^{(k)}=\frac{1}{2}\left\langle X^{(i)}, X^{(j)}\right\rangle_{t}
$$

But the left side is in $\mathfrak{M}_{c}^{l o c}$ and the right side is in $\mathfrak{A}_{c}^{l^{o c}}$. Therefore by the Corollary of Theorem 1.3, $\left\langle X^{(i)}, X^{(j)}\right\rangle_{t}=0$. Now take $h(x)=\left(x^{i}\right)^{2}-\left(x^{j}\right)^{2}$. Since this is harmonic, we see by the same argument that $\left\langle X^{(i)}\right\rangle_{t}-\left\langle X^{(j)}\right\rangle_{t} \in \mathfrak{M}_{c}^{l o c}$ $\cap \mathfrak{A}_{c}^{l o c}$ and so $\left\langle X^{(i)}\right\rangle_{t}=\left\langle X^{(j)}\right\rangle_{t}$. Thus, setting $A_{t}=\left\langle X^{(j)}\right\rangle_{t}$ we have $\left\langle X^{(i)}\right.$, $\left.X^{(j)}\right\rangle_{t}=\delta_{i, j} A_{t}$.

Now we shall prove, using the assumption (3.2) that $A_{t}$ is strictly increasing in $t$ with probability one. For simplicity we shall assume in the future $E\left(A_{t}\right)<\infty$ for every $t>0$, since the argument can be reduced to this case by truncating by stopping times. Suppose $A_{t}$ is not strictly increasing with positive probability, then for some rational $r>0$

$$
P\left(\exists \delta>0, A_{r+\delta}=A_{r}\right)>0 .
$$

This is equivalent to

$$
P\left(T_{r}>r\right)>0
$$

where $T_{r}$ is given by

$$
T_{r}=\left\{\begin{array}{c}
\inf \left\{t>r ; A_{t}>A_{r}\right\} \\
+\infty \quad \text { if }\{\quad\}=\phi .
\end{array}\right.
$$

Note that $T_{r}$ is stopping time and also $A_{T_{r}}=A_{r}$ since $A_{t}$ is continuous. Now set for $\varepsilon>0$ 


$$
S_{r}^{\varepsilon}=\left\{\begin{array}{l}
\inf \left\{t>r ;\left|X_{t}-X_{r}\right| \geqq \varepsilon\right\} \\
+\infty \quad \text { if }\{\quad\}=\phi
\end{array}\right.
$$

Then we have

$$
P\left(S_{r}^{\varepsilon} \downarrow r \text { when } \varepsilon \downarrow 0\right)=1
$$

In fact for every $\eta>r$,

$$
\begin{gathered}
\left\{S_{r}^{\varepsilon} \geqq \eta \text { for all } \varepsilon>0\right\} \underset{\text { a.s. }}{\subseteq}\left\{\left|X_{s}-X_{r}\right| \leqq \varepsilon, s \in[r, \eta], \text { for all } \varepsilon>0\right\} \\
=\left\{\left|X_{s}-X_{r}\right|=0, s \in[r, \eta]\right\}
\end{gathered}
$$

and probability of the latter set is zero by (3.2). Let $r^{\prime}>r$ then by (3.4) and (3.6) there exists $\varepsilon>0$ such that

$$
P\left(T_{r \wedge} r^{\prime}>S_{r}^{\varepsilon}\right)>0
$$

Then

$$
0=E\left(A_{T_{r} \wedge r, \wedge} S_{r}^{\varepsilon}-A_{r}\right)=\frac{1}{N} E\left(\left|X_{T_{r} \wedge r, \wedge S_{r}^{\varepsilon}}-X_{r}\right|^{2}\right) \geqq \frac{\varepsilon^{2}}{N} P\left(T_{r} \wedge r^{\prime}>S_{r}^{\varepsilon}\right)>0
$$

and this is a contradiction. Thus we have proved that with probability one $A_{t}$ is increasing.

Now set for $u>0$

$$
T_{u}=\left\{\begin{array}{ll}
\inf \{t ; & \left.A_{t}>u\right\} \\
+\infty & \text { if }\{\quad
\end{array}\right\}=\phi .
$$

Then $T_{u}$ is a stopping time $\left(\mathfrak{\mho}_{t}\right)$ and by optional sampling theorem of Doob, we have, for $s \geqq s^{\prime}$ and $u \geqq v$

$$
E\left(X_{T_{u} \wedge s}^{(i)} \mid \Im_{T_{v} \wedge s^{\prime}}\right)=X_{T_{v} \wedge s^{\prime}}^{(i)}, \quad i=1,2, \ldots \ldots, N
$$

and

$$
\begin{aligned}
& E\left(\left(X_{T_{u} \wedge s}^{(i)}-X_{T_{v} \wedge s^{\prime}}^{(i)}\right)\left(X_{T_{u} \wedge s}^{(j)}-X_{T_{u} \wedge s^{\prime}}^{(j)}\right) \mid \mho_{T_{v} \wedge s^{\prime}}\right) \\
& =\delta_{i j} E\left(A_{T_{u} \wedge s}-A_{T_{v} \wedge s^{\prime}} \mid \mho_{T_{v} \wedge s^{\prime}}\right) .
\end{aligned}
$$

Then $\tilde{X}_{u}^{(i)}=\lim _{s \rightarrow \infty} X_{T_{u} \wedge s}^{(i)}$ exists with probability one and also in $L^{2}$-sense and if we set

$$
\tilde{\mathfrak{F}}_{u}=\underset{s>0}{\bigvee} \mathfrak{F}_{u} \wedge s
$$

we have 


$$
E\left(\tilde{X}_{u}^{(i)} \mid \tilde{\mathfrak{F}}_{v}\right)=\tilde{X}_{v}^{(i)} \quad \text { a.s. }
$$

and

$$
E\left(\left(\tilde{X}_{u}^{(i)}-\tilde{X}_{v}^{(i)}\right)\left(\tilde{X}_{u}^{(j)}-\tilde{X}_{v}^{(j)}\right) \mid \tilde{\digamma}_{v}\right)=\delta_{i j} E\left(A_{\infty} \wedge u-A_{\infty} \wedge v \mid \tilde{\digamma}_{v}\right) .
$$

Thus $X_{u}^{(i)}, i=1,2, \ldots \ldots, N$ are square integrable martingales with respect to $\tilde{F}_{u}$ such that

$$
<\tilde{X}^{(i)}, \tilde{X}^{(j)}>_{u}=\delta_{i j} A_{\infty} \wedge u .
$$

Now let $\left(Y_{u}, \mathbb{E}_{u}\right)$ be an $N$-dimensional Brownian motion defined on a probability space $\left(\Omega^{\prime}, \mathfrak{E}, P^{\prime}\right)$. Let $\Omega^{*}=\Omega \times \Omega^{\prime}, \mathfrak{F}_{u}^{*}=\tilde{\mathfrak{F}}_{u} \times G_{u}, P^{*}=P \times P^{\prime}$ and define

$$
B_{u}^{(i)}=Y_{u}^{(i)}-Y_{u \wedge A_{\infty}}^{(i)}+\tilde{X}_{u}^{(i)} .
$$

For each $t, 0 \leqq t \leqq \infty, A_{t}$ is an stopping time $\left(\mathfrak{F}_{\mathfrak{u}}^{*}\right)$ since

$$
\left\{A_{t}>u\right\}=\left\{T_{u}<t\right\}=\bigcap_{n}\left\{T_{u} \wedge n<t\right\} \in \underset{n}{\bigvee} \mathscr{F}_{T_{u} \wedge n}=\tilde{F}_{u} .
$$

Then $Y_{u}^{(i)}, Y_{u \wedge}^{(i)} A_{\infty}$ and $\tilde{X}_{u}^{(i)}$ are all martingales $\left(\mathscr{F}_{u}^{*}\right)$ and so $B_{u}^{(i)}$ is a martingale $\left(\mathfrak{F}_{u}^{*}\right)$. Also we have by (3.13) that $\left\langle B^{(i)}, B^{(j)}\right\rangle_{u}=\delta_{i j}\left(u-u \wedge A_{\infty}+u \wedge A_{\infty}\right)=\delta_{i j} u$. Then by Th. 2.3, $\left(B_{u}, \mathfrak{F}_{u}^{*}\right)$ is an $N$-dimensional Brownian motion. As we have remarked above, $t \rightarrow A_{t}$ is a time change function $\left(\mathfrak{F}_{u}^{*}\right)$ and clearly

$$
X_{t}=\tilde{X}_{A_{t}}=B_{A_{t}} .
$$

This proves the theorem.

Remark. For almost all $\omega,\left\{X_{t}, t \in[0, \infty)\right\}=\left\{B_{u}, u \in\left[0, A_{\infty}\right)\right\}$. So if

$$
P\left(\sup _{t \in[0, \infty)}\left|X_{t}\right|=+\infty\right)=1
$$

then we have

$$
P\left(\lim _{t \rightarrow \infty} A_{t}=+\infty\right)=1 .
$$

\section{§ 4. Orthogonal decomposition of the space of square integrable martingales.}

I. We shall assume here that the probability space $(\Omega, \mathfrak{F}, P)$ is separable (i.e. $L^{2}(\Omega, \mathfrak{F}, P)$ is separable). We define semi-norms \|\|$_{t}$ over $\mathfrak{M}$ by the formula $\|X\|_{t}=E\left(X_{t}^{2}\right), X \in \mathfrak{M}$. Then

Proposition 4.1. $\left\{\mathfrak{M},\|\|_{t}\right\}$ is a separable complete space.

Proof. First we shall show the completeness. Let $\left\{X^{n}\right\}$ be a Cauchy 
sequence in $\mathfrak{M}$. Since $\left\{X^{n}\right\}$ are martingales, by Doob's inequality [2, Chap. VII, Theorem 3. 2] we have

$$
P\left(\sup _{0<s<t}\left|X_{s}^{n}-X_{s}^{m}\right| \geqq c\right) \leqq 1 / c^{2} E\left(\left(X_{t}^{n}-X_{t}^{m}\right)^{2}\right) \underset{n, m \rightarrow \infty}{\rightarrow} 0
$$

for every $c>0$. Therefore $X_{t}^{n}$ converges locally uniformly to $X_{t}$ a.e.P. It is clear that $X$ is an element of $\mathfrak{M}$ and satisfies $\left\|X-X^{n}\right\|_{t} \rightarrow 0$ as $n \rightarrow \infty$. The uniqueness of such $X_{t}$ is also clear. Next, we will show the separability. Let $L_{2}^{0}(P)$ be the space of all $\mathfrak{F}$-measurable functions $f$ such that $E\left(f^{2}\right)<\infty$ and $E(f)=0$. Let $X_{t}^{f}$ be the right continuous version of $E\left(f / \mathfrak{F}_{t}\right)$ and $\mathfrak{M}^{0}=\left\{X_{t}^{f}\right.$; $\left.f \in L_{2}^{0}(P)\right\}$. Then $\mathfrak{M}^{0}$ is dense in $\left\{\mathfrak{M},\|\|_{t}\right\}$. Let $\left\{f^{n}\right\}$ be a dense sequence of $L_{2}^{0}(P)$. Then $\left\{X_{t}^{f n}\right\}$ are dense in $\mathfrak{M}^{0}$. Indeed, let $f$ be any element of $L_{2}^{0}(P)$ and $\left\{f^{n i}\right\}$ a sequence converging to $f$ in $L_{2}^{0}(P)$ space. Then, by Jensen's inequality we have

$$
E\left(\left(X_{t}^{f}-X_{t}^{\left.f_{n i}\right)^{2}}\right) \leqq E\left(\left(f-f^{n i}\right)^{2}\right) \underset{n_{i} \rightarrow \infty}{\rightarrow} 0\right.
$$

Therefore $\left\{X^{f_{n i}}\right\}$ converges to $X^{f}$.

Definition 4.1. $\quad X, Y \in \mathfrak{M}$ are called orthogonal and denoted by $X \perp Y$ if and only if $\langle X, Y\rangle=0$.

Meyer defined that $X, Y \in \mathfrak{M}$ are orthogonal if $X_{t} Y_{t}$ is a martingale. Our definition is the same as Meyer's one. Indeed, since

$$
E\left(\left(X_{t}-X_{s}\right)\left(Y_{t}-Y_{s}\right) / F_{s}\right)=E\left(X_{t} Y_{t} / F_{s}\right)-X_{s} Y_{s},
$$

$\langle X, Y\rangle \equiv 0$ if and only if $X_{t} Y_{t}$ is a martingale.

Definition 4.2. A subset $\mathfrak{R}$ of $\mathfrak{M}$ is called a subspace of $\mathfrak{M}$ if $\mathfrak{N}$ satisfies the following three conditions;

(i) $X, Y \in \mathfrak{N} \rightarrow X+Y \in \mathfrak{N}, \quad$ (ii) if $X \in \mathfrak{N}$ and $\Phi \in \Phi(\langle X\rangle)$, then $\int \Phi d X \in \mathfrak{N}$ and (iii) $\mathfrak{R}$ is closed in $\left\{\mathfrak{M},\|\quad\|_{t}\right\}$.

The smallest subspace containing $\mathfrak{N}(\subset \mathfrak{M})$ is denoted by $\mathfrak{L}(\mathfrak{N})$ and is said to be generated by $\mathfrak{R}$. It is easy to see $\mathfrak{L}(X)=\left\{\int \Phi d X ; \Phi \in \Phi(\langle X\rangle)\right\}$.

Let $\mathfrak{R}$ be a subset of $\mathfrak{M}$ and set $\mathfrak{R}^{\perp}=\{Y \in \mathfrak{M} ; Y$ is orthogonal to every $X$ of $\mathfrak{R}\}$. We can easily show that $\mathfrak{R}$ is a subspace, $\mathfrak{L}(\mathfrak{R})^{\perp}=\mathfrak{R}^{\perp}$ and $\mathfrak{L}(\mathfrak{R}) \cap \mathfrak{R} \mathfrak{R}^{\perp}=\{0\}$.

PROPOSITION 4.1. Let $X$ and $Y$ be elements of $\mathfrak{M}$. There exist unique $Y \in \mathfrak{R}(X)$ and $Y^{\prime \prime} \perp \mathfrak{L}(X)$ such that $Y=Y^{\prime}+Y^{\prime \prime}$. 
Proof. First we shall note that $\langle X, Y\rangle_{t}$ is absolutely continuous with respect to $\langle X\rangle$ in the following sense. If $\Phi \in \Phi(\langle X\rangle)$ satisfies $\Phi=\Phi^{2}$ and $\int \Phi d\langle X\rangle=0$, then $\int \Phi d\langle X, Y\rangle=0$. For, since $\int \Phi d\langle X\rangle=0$ implies $\int \Phi d X=0$ we have $\int \Phi d\langle X, Y\rangle=\left\langle\int \Phi d X, Y\right\rangle=0$, which implies the absolute continuity. Therefore, we can choose $\Phi \in \Phi(\langle X, Y\rangle)$ such that $\langle X, Y\rangle_{t}=\int_{0}^{t} \Phi d\langle X\rangle_{s}$. Set $Y^{\prime}=\int \Phi d X$ and $Y^{\prime \prime}=Y-Y^{\prime}$. Then $\left\langle X, Y^{\prime \prime}\right\rangle=\langle X, Y\rangle-\left\langle X, Y^{\prime}\right\rangle=0$, showing that $X \perp Y^{\prime \prime}$. The uniqueness is clear.

Definition 4.3. $\quad Y^{\prime}$ is called the projection of $Y$ to the space $\mathfrak{L}(X)$ and is denoted by $P_{\mathfrak{R}(X)} Y$.

Let $\left\{X^{n}\right\}$ be a sequence of $\mathfrak{M}$. We can choose a sequence $\left\{Y^{n}\right\}$ such that these are mutually orthogonal and $\mathfrak{L}\left(\left\{X^{n}\right\}\right)=\mathfrak{L}\left(\left\{Y^{n}\right\}\right)$. The proof is due to usual Schmidt's orthogonalization method. Indeed, define $Y^{n}$ by induction as $Y^{n}=X^{n}-\sum_{i=1}^{n-1} P_{\mathfrak{Q}\left(Y^{i}\right)} X^{n} . \quad$ Then it turns out that $\left\{Y^{n}\right\}$ are mutually orthogonal and $\mathfrak{L}\left(\left\{Y^{n}\right\}\right)=\mathfrak{L}\left(\left\{X^{n}\right\}\right)$. Now let $Z$ be any element of $\mathfrak{M}$. Then $\left\{\sum_{i=1}^{n} P_{\mathfrak{R}\left(Y^{i}\right)} Z\right\}$ is a Cauchy sequence in $\left\{\mathfrak{M},\|\quad\|_{t}\right\}$. For since $Z^{n} \perp \sum_{i=1}^{n} P_{\mathfrak{R}\left(Y^{i}\right)}$, we have

$$
\langle Z\rangle=\left\langle Z^{n}\right\rangle+\sum_{i=1}^{n}\left\langle P_{\mathfrak{L}\left(Y^{i}\right)} Z\right\rangle
$$

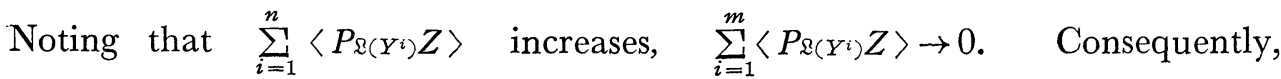
$\left\{\sum_{i=1}^{n} P_{\mathfrak{R}\left(Y^{i}\right)} Z\right\}$ is a Cauchy sequence. Define $Z^{\prime}=\lim _{n \rightarrow \infty} \sum_{i=1}^{n} P_{\mathfrak{L}\left(Y^{i}\right)} Z$ and $Z^{\prime \prime}=Z-Z^{\prime}$. Then $Z^{\prime \prime}$ is orthogonal to $\mathfrak{R}\left(\left\{Y^{n}\right\}\right)$. Thus we have proved

Theorem 4.1. (i) Let $\mathfrak{N}$ be a subspace of $\mathfrak{M}$. There is a sequence $\left\{Y^{n}\right\}$ such that they are mutually orthogonal and $\mathfrak{L}\left(\left\{Y^{n}\right\}\right)=\mathfrak{R}$. (ii) Any element of $\mathfrak{M}$ can be decomposed uniquely to $Z=Z^{\prime}+Z^{\prime \prime}$ where $Z^{\prime} \in \mathfrak{N}$ and $Z^{\prime \prime} \in \mathfrak{N} \perp$. Furthermore, $Z^{\prime}=\lim _{n \rightarrow \infty} \sum_{i=1}^{n} P_{\mathfrak{s}\left(Y^{i}\right)} Z$.

Definition 4.4. $\quad Z^{\prime}$ is called the projection of $Z$ to the space $\Re$ and is denoted by $P_{\Re} Z$.

Corollary. $\quad\left(\mathfrak{R}^{\perp}\right)^{\perp}=\mathfrak{R}$.

Proof. It is clear that $\left(\mathfrak{R}^{\perp}\right)^{\perp} \supset \mathfrak{R}$. Let $Z$ be any element of $\left(\mathfrak{R}^{\perp}\right)^{\perp}$. We have only to prove $Z=P_{\mathfrak{n}} Z$. Since $Z-P_{\mathfrak{N}} Z \in \mathfrak{R}^{\perp} \cap\left(\mathfrak{R}^{\perp}\right)^{\perp}$, we have $Z=P_{\mathfrak{n}} Z$. 
II. Case of Markov processes. We shall discuss further detailed structure of the square integrable martingales $\mathfrak{M}$ when the underlying probability space is that of a Hunt process. As a result, it turns out that $\mathfrak{M}$ is generated by additive functionals of the Hunt process.

Throughout this and the following sections, we treat the case of Hunt process. So we shall give here the definition of Hunt process. Let $S$ be a locally compact Hausdorff space with a countable base and $\infty$ a point adjoined to $S$ as one point compactification. Set $S^{*}=S \cup\{\infty\}$. The topological $\sigma$-algebra of $S$ is denoted by $B_{S}$ and the set of all bounded $B_{S}$-measurable functions is denoted by $B(S)$. Let $(\Omega, \mathfrak{F})$ be a basic measurable space and $x_{t}(\omega)$, a measurable mapping from $[0, \infty) \times \Omega$ to $S^{*}$ such that it is right continuous and has left hand limits with respect to $t$ and $x_{t}(\omega)=\infty$ for $t \geqq \zeta(\omega)=\inf \left\{t>0 ; x_{t}(\omega)=\infty\right\}$. The shift operator $\theta_{t}$ is defined as $x_{s}\left(\theta_{t} \omega\right)=x_{t+s}(\omega) . \mathfrak{B}_{t}$ is a $\sigma$-subalgebra of $\mathfrak{F}$ generated by the set $\left\{x_{s}(\omega) \in E\right\}\left(s \leqq t, E \in B_{S}\right)$. Let $P_{x}, x \in S$, be a family of probability measures on $(\Omega, \mathfrak{F})$ such that $P_{x}(B), B \in \mathfrak{B}_{t}$ is $B_{S}$-measurable and $P_{x}\left(x_{0}(\omega)\right.$ $=x)=1$. Set $\mathfrak{F}_{\infty}=\cap P_{\mu}$-completion of $\mathfrak{B}_{\infty}$, where $P_{\mu}=\int \mu(d x) P_{x}$ and $\mu$ runs all bounded Radon measure over $S$, and $\mathfrak{F}_{t}=\left\{B \in \mathfrak{F}_{\infty} ; \forall \mu, \exists B_{\mu} \in \mathfrak{B}_{t}\right.$ such that $P \mu(B \Delta B \mu)=0\} . \quad M=\left(x_{t}, \zeta, \mathfrak{F}_{t}, P_{x}\right)$ is called a Hunt process if each $P_{x}$ has the strong Markov property and quasi-left continuity of sample paths, i.e., for every increasing sequence of stopping times $\left\{T_{n}\right\}$ converging to $T, x_{T_{n}}$ converges to $x_{T}$ on the set $\{T<\infty\}$ a. s. $P_{x}$. It is well known that $\mathfrak{F}_{t}$ has no time of discontinuity.

Under this definition of Hunt process, definitions and arguments such as $\S \S 1-3$ and the first part of this section require more careful treatment, because the family of measures $P_{x}, x \in S$ is given on $\left(\Omega, \mathfrak{F}_{t}\right)$. Every fact concerning the measure $P$ should be replaced to the fact concerning the family of measures $P_{x}, x \in S$.

$A$ real valued function $X_{t}(\omega), t \in[0, \infty), \omega \in \Omega$ is called a functional if it is $\mathfrak{F}_{t}$-measurable for each $t$ and is right continuous a.e. $P_{x}(\forall x \in S)$. A functional $X_{t}$ is called a martingale if $E_{x}\left(\left|X_{t}\right|\right)<\infty$ and $E_{x}\left(X_{t} / \mho_{s}\right)=X_{s}$ a.e. $P_{x}(\forall x)$. $\mathfrak{M}$ is the space of all martingales such that $E_{x}\left(X_{t}^{2}\right)<\infty$ for every $t>0$ and $X \in S$ and that $E_{x}\left(X_{t}\right)=0$ for every $x \in S$. $\mathfrak{Q}^{+}$is the family of natural increasing processes (and also functionals) $A_{t}$ such that $E_{x}\left(A_{t}\right)<\infty$ for $t>0$ and $x \in S$. The definitions of $\mathfrak{M}^{l o c}$ and $\mathfrak{A}$ are made in a similar way. We introduce semi-norms \|\|$\|_{x, t}$ over $\mathfrak{M}$ by the formula $\|X\|_{x, t}=E_{x}\left(X_{t}^{2}\right)$. Then

Proposition 4.1'. $\quad\left\{M \mathbb{R},\|\|_{x, t}\right\}$ is complete. 
Proof. Let $\left\{X^{n}\right\}$ be a Cauchy sequence. Set $e_{n, m}(t, x)=\left\|X^{n}-X^{m}\right\|_{t, x}$ and define

$$
n_{i}(x)=\min \left\{n ; \sup _{\substack{m \geq n \\ m^{\prime} \geq n}} e_{m, m^{\prime}}(i, x)<1 / 3^{2 i}\right\}, \quad i=1,2, \ldots \ldots
$$

Then $n_{i}(x)$ is $B_{S}$-measurable for each $i$ and so $n_{i}\left(x_{0}(\omega)\right)$ is $\Re_{\infty}$-measurable. Since

$$
\left\|\left(X^{n_{i}\left(x_{\oplus}\right)}-X^{n_{j}\left(x_{\diamond}\right)}\right)\right\|_{t, x} \leqq 1 / 3^{2 i} \text { for each } x \text { and } t \leq i \leq j,
$$

we have by a martingale inequality

$$
P_{x}\left(\max _{0 \leq s \leq k}\left|X_{s}^{n_{i}\left(x_{\odot}\right)}-X_{s}^{n_{f}\left(x_{\theta}\right)}\right|>1 / 2^{i}\right) \leqq(2 / 3)^{2 i}
$$

for every $x$ and $k \leqq i \leq j$. Set $\widetilde{\Omega}=\left\{\omega ; X_{s}^{n_{i}\left(x_{\theta}(\omega)\right)}(\omega)\right.$ converges uniformly on any bounded interval in $[0, \infty)\}$, then by Borel-Cantelli's lemma $P_{x}(\widetilde{\Omega})=1$ for every $x$. For $\omega \in \widetilde{\Omega}$ define $X_{s}(\omega)=\lim _{i=\infty} X_{s}^{n_{i}\left(x_{\ominus}\right)}(\omega)$, then $X \in \mathfrak{M}$ and $X_{n}$ converges to $X$. The uniqueness of $X$ is clear.

Theorem 1.1 is modified in the following way.

Theorem 1.1'. For $X, Y \in \mathfrak{M}$, there is a unique $\langle X, Y\rangle \in \mathfrak{A}_{c}$ such that

$$
E_{x}\left(\left(X_{t}-X_{s}\right)\left(Y_{t}-Y_{s}\right) / \mathfrak{F}_{s}\right)=E_{x}\left(\langle X, Y\rangle_{t}-\langle X, Y\rangle_{s} / \mathfrak{F}_{s}\right) \text { for every } x \in S \text {. }
$$

Proof. The proof of Theorem 1.1 is based on Meyer's decomposition theorem $[8,9]$. Let $Z_{t}$ be a supermartingale relative to $\left(\mathfrak{F}_{t}, P_{x}\right)$ belonging to the class $(D)$ and $\lim _{t \rightarrow \infty} Z_{t}=0$ relative to $P_{x}$ for every $x \in S$. By Meyer's decomposition there is an increasing process $A_{t}^{(x)}$ such that $Z_{t}=E_{x}\left(A_{\infty}^{(x)} \mid \mathfrak{\mho}_{t}\right)-A_{t}^{(x)}$ for each $t$. We can prove that there is a functional $A_{t}$ such that $A_{t}=A_{t}^{(x)}$ a.e. $P_{x}$, i.e. $Z_{t}=E_{x}\left(A_{\infty} / \mathfrak{F}_{t}\right)-A_{t}$. This argument will be treated in Appendix. Using this fact, the proof of this theorem is just the same as that of Theorem 1.1.

Let $\Phi$ and $\Phi_{r c}$ be the spaces of processes defined in $\S 2$. Define semi-norms by $\|\Phi\|\langle X\rangle, x(t)=E_{x}\left(\int_{0}^{t} \Phi_{s}^{2} d\langle X\rangle_{s}\right)^{1 / 2}$. Let $L(\langle X\rangle)$ be the intersection of $\Phi$ and the completion of $\Phi_{r c}$ with respect to semi-norms $\|\quad\|<X>, x(t)$. The conclusion of Lemma 2.1. is still valid if we take $P_{x},\|\|<X>, x(t)$ for $P,\|\quad\|<X>(t)$. The proof is the same as that of Lemma 2.1. Using this fact, we can prove

Theorem 1.2'. For $X \in \mathfrak{M}$ and $\Phi \in L(\langle X\rangle)$, there is a unique $Y \in \mathfrak{M}$ satisfying

$$
\langle Y, Z\rangle_{t}=\int_{0}^{t} \Phi_{s} d\langle X, Z\rangle_{s} \text { a.e. } P_{x} \text { for every } Z \in \mathfrak{M}
$$


$A$ subspace $\mathfrak{R}$ is defined in a similar way. Also closed set is defined by the topology \|\|$_{t, x}$ in this case. Propositions and theorems in Part I are also valid and the proof is quite similar.

A functional $X_{t}$ is called an additive functional (a.f.) if it satisfies $X_{t}(\omega)+X_{s}\left(\theta_{t} \omega\right)$ $=X_{t+s}(\omega)$ for all $t, s>0$ a.e. $P_{x}$. The set of all a. $f^{\prime} . \mathrm{s}$ is denoted by A. Let $f \in B(S)$ and $u=G_{\alpha} f=E .\left(\int_{0}^{\infty} e^{-\alpha t} f\left(x_{t}\right) d t\right)$ and define

$$
X_{t}^{f, \alpha}=u\left(x_{t}\right)-u\left(x_{0}\right)-\int_{0}^{t}\left(\alpha u\left(x_{s}\right)-f\left(x_{s}\right)\right) d s .
$$

Then $X_{t}^{f,{ }^{\alpha}}$ is a square integrable a.f. . Since $E_{x}\left(X_{t}^{f, \alpha}\right)=0$,

$$
E_{x}\left(X_{t+s}^{f, \alpha} / \mathfrak{F}_{t}\right)=X_{t}^{f, \alpha}+E_{x}\left(X_{s}^{f, \alpha}\left(\theta_{t} \omega\right) / \mathfrak{F}_{t}\right)=X_{t}^{f, \alpha}+E_{x_{t}}\left(X_{s}^{f, \alpha}\right)=X_{t}^{f, \alpha} .
$$

Therefore $X_{t}^{f, \alpha} \in \mathfrak{M}$.

Theorem 4.2. ${ }^{6)} \quad\left\{X^{f, \alpha} ; f \in B(S), \alpha>0\right\}$ generates $\mathfrak{M}$.

Before the proof we prepare the following

Lemma 4.1. (i) If $X_{t}$ is of $\mathfrak{M}$, then so is

$$
X_{t}^{\alpha}=e^{-\alpha t} X_{t}+\alpha \int_{0}^{t} e^{-\alpha s} X_{s} d s
$$

(ii) If $X \in Y$ then $X^{\alpha} \in Y$. (iii) If $X^{f, \alpha}$ is of the form (4.1) then

$$
X^{f, \alpha, \alpha}=e^{-\alpha t} u\left(x_{t}\right)-u\left(x_{0}\right)+\int_{0}^{t} e^{-\alpha s} f\left(x_{s}\right) d s .
$$

The proof can be obtained by elementary calculations and it is omitted.

Proof of theorem 4.1. Suppose $Y \perp X^{f, \alpha}$ for all $f \in B(S)$ and $\alpha>0$. Then We shall show first

$$
E\left(f\left(x_{u+s}\right)\left(Y_{u+s}-Y_{s}\right) / \Re_{s}\right)=0
$$

for all $f \in B(S)$. From Lemma 4.1, we have for any $\alpha>0$,

$$
E .\left(\left(X_{t}^{f, \alpha, \alpha}-X_{s}^{f, \alpha, \alpha}\right)\left(Y_{t}-Y_{s}\right) / \mathfrak{F}_{s}\right)=0 \text {. }
$$

On the other hand since

$$
E .\left(\left(X_{\infty}^{f, \alpha, \alpha}-X_{t}^{f, \alpha, \alpha}\right)\left(Y_{t}-Y_{s}\right) / \mho_{s}\right)=0
$$

we have

$$
E .\left(\left(X_{\infty}^{f, \alpha, \alpha}-X_{s}^{f, \alpha, \alpha}\right)\left(Y_{t}-Y_{s}\right) / \mathfrak{F}_{s}\right)=0 \text {. }
$$

Noting the relation

6) c.f. Motoo, Watanabe [10]. 


$$
X_{\infty}^{f, \alpha, \alpha}-X_{s}^{f, \alpha, \alpha}=-e^{-\alpha s} u\left(x_{s}\right)+\int_{s}^{\infty} e^{-\alpha u} f\left(x_{u}\right) d u
$$

the left hand of (4.5) is

$$
\begin{aligned}
& E\left(\int_{s}^{\infty} e^{-\alpha u} f\left(x_{u}\right) d u \cdot\left(Y_{t}-Y_{s}\right) / \mathfrak{F}_{s}\right) \\
= & E\left(\int_{0}^{\infty} e^{-\alpha u} f\left(x_{u}\right)\left(E\left(Y_{t} / \mathfrak{F}_{u}\right)-Y_{s}\right) d u / \mathfrak{F}_{s}\right) \\
= & E\left(\int_{0}^{\infty} e^{-\alpha u} f\left(x_{u}\right)\left(Y_{u}^{\prime}-Y_{s}\right) d u / \mathfrak{F}_{s}\right) \\
= & E\left(\int_{0}^{\infty} e^{-\alpha u} f\left(x_{u+s}\right)\left(Y_{u+s}^{\prime}-Y_{s}\right) d u / \mathfrak{F}_{s}\right),
\end{aligned}
$$

where $Y_{u}^{\prime}=Y_{u \wedge t}$. Therefore from (4.5) and (4.6) we have

$$
E .\left(f\left(x_{u+s}\right)\left(Y_{u+s}^{\prime}-Y_{s}\right) / \mathfrak{F}_{s}\right)=0
$$

and since $t$ is arbitrary we have (4.4). Now let $0=t_{0}<t_{1}<t_{2}<\ldots \ldots<t_{n}$ and $f_{1}, \ldots \ldots, f_{n} \in B(S)$. Using the Markov property and noting (4.4),

$$
\begin{gathered}
E .\left(f_{1}\left(x_{t_{1}}\right) \ldots \ldots f_{n}\left(x_{t_{n}}\right) Y_{t_{n}}\right)=\sum_{j=0}^{n-1} E .\left(f_{1}\left(x_{t_{1}}\right) \ldots \ldots f_{n}\left(x_{t_{n}}\right)\left(Y_{t_{j+1}}-Y_{t_{j}}\right)\right) \\
=\sum_{j=0}^{n-1} E .\left(f_{1}\left(x_{t_{1}}\right) \ldots \ldots f_{j+1}\left(x_{t_{j+1}}\right) E_{x_{t_{j+1}}}\left(f_{j+2}\left(x_{t_{j+2}-t_{j+1}}\right) \ldots \ldots f_{n}\left(x_{t_{n}-t_{j+1}}\right)\right)\left(Y_{t_{j+1}}-Y t_{j}\right)\right)=0,
\end{gathered}
$$

which implies $Y_{t_{n}}=0$. Therefore $Y_{t} \equiv 0$.

Corollary. $\mathfrak{M} \cap \forall$ generates $\mathfrak{M}$ and $\mathfrak{M}_{c} \cap \forall$ generates $\mathfrak{M}_{c}$.

ExAmple. Let $x_{t}$ be an $N$-dimensional Brownian motion; $x_{t}=\left(B_{t}^{(1)}, \ldots \ldots\right.$, $\left.B_{t}^{(N)}\right)$. Then $\alpha u-f=\frac{1}{2} \Delta u$. The formula on stochastic integral implies together with this relation that $X_{t}^{f, \alpha}$ defined by (4.1) satisfies

$$
X_{t}^{f, \alpha}=\sum_{i=1}^{N} \int \frac{\partial u}{\partial x^{i}}\left(x_{s}\right) d B_{s}^{(i)}
$$

Therefore $\mathfrak{M} \mathcal{M}=\mathfrak{Q}\left(X_{t}^{f, \alpha} ; f \in B(S)\right)=\mathfrak{L}\left(B_{t}^{(1)}, \ldots \ldots, B_{t}^{(N)}\right)$. This fact together with Theorem 4.1 shows that every $X$ of $\mathfrak{M}$ is represented as $X_{t}=\sum_{i=1}^{N} \int_{0}^{t} \Phi_{s}^{i} d B_{s}^{(i)}$.

\section{$\S 5$. Formula on stochastic integrals. II}

Let us define $\mathfrak{M}_{d}$ as the orthogonal complement of the subspace $\mathfrak{M}_{c}$. We will first discuss the structure of $\mathfrak{M i}_{d}$. Let $\rho$ be a metric on $S$. Set for convenience $\rho(x,\{\infty\})=\infty$ for every $x \in S$ and $\chi_{\varepsilon}(x, y)=\chi_{\{\rho(x, y)>\varepsilon\}}(x, y)$. Define $P_{\varepsilon}(t, E)$ $\sum_{S \leq t} \chi_{\varepsilon}\left(x_{s-}, x_{s}\right) \chi_{E}\left(x_{s}\right),{ }^{7)} E \in B_{S}$, and $\Gamma^{\varepsilon}=\left\{E \in B_{S} ; E_{x}\left(P_{\varepsilon}(t, E)\right)<\infty\right.$ for every $t>0$

7) $x_{s-}=\lim _{h \downarrow 0} x_{s-h}$ 
and $x \in S\}$. It is known in [11] that there exists a non-negative continuous a.f. $\varphi_{t}^{0}$ and a kernel $n(x, d y)$ such that

$$
q_{\varepsilon}(t, E)=P_{\varepsilon}(t, E)-\int_{0}^{t}\left\{\int_{E} \chi_{\varepsilon}\left(x_{s}, y\right) n\left(x_{s}, d y\right)\right\} d \varphi_{s}^{0}, E \in \Gamma_{\varepsilon}
$$

is a square integrable martingale. The system $\left(n(x, d y), \varphi_{t}^{0}\right)$ is called a Levy system. Further $q \varepsilon(t, E)$ and $q_{\varepsilon^{\prime}}(t, F)$ satisfy

$$
\left\langle q_{\varepsilon}(t, E), q_{\varepsilon^{\prime}}(t, F)\right\rangle=\int_{0}^{t}\left\{\int_{E \cap F^{\prime}} \chi_{\varepsilon \vee \varepsilon^{\prime}}\left(x_{s}, y\right) n\left(x_{s}, d y\right)\right\} d \varphi_{s}^{0} .
$$

Let $f(t, y, \omega)$ be a $B_{[0, \infty)} \times B_{S} \times \mathfrak{F}_{\infty}$-measurable function such that $f(t, y, \omega) \in$ $L\left(\varphi^{0}\right)$ for any fixed $y$. Set

$$
\begin{aligned}
& \|f\|_{Q}^{t, x}=E_{x}\left(\int_{0}^{t}\left\{\int_{S} f(s, y, \cdot)^{2} n\left(x_{s}, d y\right)\right\} d \varphi_{s}^{0}\right)^{1 / 2} \\
& F_{Q}=\left\{f ;\|f\|_{Q}^{t, x}<\infty \text { for every } t>0 \text { and } x \in S\right\} .
\end{aligned}
$$

Proposition 5.1. For evbry $f \in F_{Q}$, we can associate $Q_{r}(t)$ of $M d$ denoted by $\int_{0}^{t} \int_{S} f(s, y, \cdot) q(d s, d y)$ in such a way that (i) if

$$
f(s, y, \cdot)=\Phi_{s} \chi_{\varepsilon}\left(x_{s}, y\right) \chi_{E}(y), \quad \Phi \in L\left(<q_{\varepsilon}(., E)>\right), \varepsilon>0, E \in \Gamma^{\varepsilon},
$$

then

$$
Q_{f}(t)=\int_{0}^{t} \Phi_{s} q_{\varepsilon}(d s, E),
$$

(ii) if $f, g \in F_{Q}$ and $a, b \in R^{1}$, then

$$
Q_{a f+b g}=a Q_{f}+b Q_{g} .
$$

(iii) $\left\|Q_{f}\right\|_{t, x}=\|f\|_{Q}^{t, x}$.

Furthermore, $Q_{f}$ is uniquely determined by these properties.

Proof. If $f$ is of the form (5.3), define $Q_{f}$ by (5.4). When $f=\sum_{i=1}^{n} f^{i}$ and $f^{i}=\Phi_{s}^{i} \chi_{\varepsilon_{i}}\left(x_{s}, y\right) \chi_{E_{i}}(y)$, define $Q_{f}=\sum_{i=1}^{n} Q_{f^{i}}$. By the definition of stochastic integral and (5.2)

$$
\begin{aligned}
<Q_{f^{i}}, Q_{f^{j}}>_{t} & =\int_{0}^{t} \Phi_{s}^{i} \Phi_{s}^{j} d<Q_{\varepsilon_{i}}\left(\cdot, E_{i}\right), q_{\varepsilon_{j}}\left(\cdot, E_{j}\right)>_{s} \\
& =\int_{0}^{t}\left\{\int_{E_{i} \cap E_{j}} \Phi_{s}^{i} \Phi_{s}^{j} \chi_{\varepsilon_{i} \vee \varepsilon_{j}}\left(x_{s}, y\right) n\left(x_{s}, d y\right)\right\} d \varphi_{s}^{0} \\
& =\int_{0}^{t}\left\{\int_{S} f^{i} f^{j} n\left(x_{s}, d y\right)\right\} d \varphi_{s}^{0}
\end{aligned}
$$


and so

$$
\left\|Q_{f}\right\|^{t, x}=\sum_{i, j=1}^{n} E_{x}\left(<Q_{f^{i}}, Q_{f^{j}}>_{t}\right)=\|f\|_{Q}^{t, x}
$$

Let $f$ be any element of $F_{Q}$. We can choose $\left\{f^{n}\right\}$ such that each $f^{n}$ is a linear combination of the form (5.3) and $\left\|f-f^{n}\right\|_{Q}^{t, x} \rightarrow 0$. Then $\left\{Q_{f^{n}}\right.$ is a Cauchy sequence by (5.6) and $Q_{f}$ defined by $\lim _{n \rightarrow \infty} Q_{f^{n}}$ is of $\mathfrak{M}_{d}$. It is clear that (ii) and (iii) are satisfied by $f$ for this $Q_{f}$. The uniqueness will be obvious.

Proposition 5.2. $\quad \mathfrak{M}_{d}=\left\{Q_{f} ; f \in F_{Q}\right\}$.

Proof. By the above proposition, it is clear that $\left\{Q_{f} ; f \in F_{Q}\right\}=\Omega\left\{q_{\varepsilon}(\cdot, E)\right.$; $\left.\varepsilon>0, E \in \Gamma_{\varepsilon}\right\}$. On the other hand it is known in [9] that

$$
\mathfrak{M}_{d} \cap \boldsymbol{A}=\mathfrak{L}\left(q_{\varepsilon}(\cdot, E) ; \varepsilon>0, E \in \Gamma_{\varepsilon}\right) \cap \boldsymbol{A} .
$$

Therefore we get the proposition.

The orthogonality of $X, Y \in \mathfrak{M}^{l o c}$ is also defined by $\langle X, Y\rangle=0$. Many arguments in the preceding sections and this section can be extended to $\mathfrak{M}^{l o c}$. Let $\mathfrak{M}_{d}^{l o c}$ be the orthogonal complement of $\mathfrak{M}_{c}^{l o c}$. We will call $f(t, y, \omega)$ belongs to $F_{Q}^{l o c}$ if there exists a sequence of stopping times $\left\{T_{n}\right\}$ converging to $\infty$ such that

$$
E\left(\int_{0}^{T_{n} \wedge t}\left\{\int_{S} f(s, y, \omega)^{2} n\left(x_{s}, b y\right)\right\} d \varphi_{s}^{0}\right)<\infty, \quad n=1,2, \ldots \ldots
$$

The stochastic integral $Q_{f}(t) \in \mathfrak{M}_{d}^{l o c}, f \in F_{Q}^{l o c}$ is defined similarly as in Proposition 6.1 and we have $\mathfrak{M}_{d}^{\text {loc }}=\left\{Q_{f} ; f \in F_{Q}^{\text {loc }}\right\}$.

$A$ functional $\varphi_{t}$ is called quasi-left continuous (q.1.c.) if it has jumps only at the discontinuity points of sample path. Let $\varphi_{t}$ be a purely discontinuous q.1.c. functional. There exists a $(s, y, \omega)$-measurable function $f(s, y, \omega)$ such that $\varphi_{t}(\omega)=\sum_{\rho\left(x_{s}, x_{s-}\right)>0, s \leqq t} f\left(s, x_{s}, \omega\right)$. We shall write such $\varphi_{t}$ as $P_{f}(t)=\int_{0}^{t} \int_{S} f(s, y, \omega) P(d s, d y)$.

Theorem 5.1. Let $F$ be a $C^{2}$-class function on $R^{N}$. Let $X^{(i)} \in \mathfrak{M}_{c}^{l o c}$, $Q_{f}^{(i)} \in \mathfrak{M}_{d}^{l o c}, \varphi_{t}^{(i)} \in \mathfrak{A}_{c}^{l o c}$ and $P_{g^{(i)}(t)}$ be a purely discontinuous q.1.c. functional such that $f^{(i)} \cdot g^{(i)}=0$. Set $A_{t}^{(i)}=X_{t}^{(i)}+Q_{f}^{(i)}(t)+\varphi_{t}^{(i)}+P_{g}^{(i)}(t), \quad$ and $\boldsymbol{f}=\left(f^{(1)}, \ldots \ldots, f^{(N)}\right) \boldsymbol{g}$ $=\left(g^{(i)}, \ldots \ldots, g^{(N)}\right), \boldsymbol{A}_{t}=\left(A_{t}^{(i)}, \ldots \ldots, A_{t}^{(N)}\right)$. Suppose that one of the following conditions is satisfied; (i) Each $\left|\frac{\partial F}{\partial x^{i}}\right|$ is bounded, (ii) each $f^{(i)}$ is bounded. Then the following formula is satisfied 


$$
\begin{aligned}
& F\left(\boldsymbol{A}_{t}\right)-F\left(\boldsymbol{A}_{0}\right)=\sum_{i=1}^{N} \int_{0}^{t} \frac{\partial F}{\partial x^{i}}\left(\boldsymbol{A}_{s}\right) d X_{s}^{(i)}+\int_{0}^{t} \int_{S}\left(F\left(\boldsymbol{A}_{s}+\boldsymbol{f}(s, y, \cdot)\right)-F\left(\boldsymbol{A}_{s}\right)\right) q(d s, d y) \\
& \left.+\int_{0}^{t} \int_{S}\left\{F\left(\boldsymbol{A}_{s}+\boldsymbol{f}(s, y, \cdot)\right)-F\left(\boldsymbol{A}_{s}\right)-\sum_{i=1}^{N} f(s, y, \cdot) \frac{\partial F}{\partial x^{i}}\left(\boldsymbol{A}_{s}\right)\right)\right\} n\left(x_{s}, d y d \varphi_{s}^{0}\right. \\
& +P_{G}(t)+\sum_{i=1}^{N} \int_{0}^{t} \frac{\partial F}{\partial x^{i}}\left(\boldsymbol{A}_{s}\right) d \varphi_{s}^{(i)}+\frac{1}{2} \sum_{i, j=1}^{N} \int_{0}^{t} \frac{\partial^{2} F}{\partial x^{i} \partial x^{j}}\left(\boldsymbol{A}_{s}\right) d\left\langle X^{(i)}, X^{(j)}\right\rangle_{s}
\end{aligned}
$$

where $G(s, y, \omega))=F\left(\boldsymbol{A}_{s}+\boldsymbol{g}(s, y, \omega)\right)-F\left(\boldsymbol{A}_{s}\right)$.

Proof. We shall consider the case $N=1$ for simplicity. Assume that $f(s, y, \omega)=\chi_{\varepsilon}\left(x_{s-}, y\right) g(s, y, \omega)$ and $g(s, y, \omega)=\chi_{\varepsilon}\left(x_{s-}, y\right) g(s, y, \omega)$. Define $T_{0}=0$ and

$$
T_{n}=\inf \left\{t>T_{n-1} ; \rho\left(x_{t-}, x_{t}\right)>\varepsilon\right\} .
$$

Then $T_{n} \uparrow \infty$ as $n \rightarrow \infty$ and jumps of $Q_{f}$ and $P_{g}$ occur only at $\left\{T_{n}\right\}$. Therefore, using Theorem 2.2,

$$
\begin{aligned}
& F\left(A_{T_{n} \wedge t-}\right)-F\left(A_{T_{n-1} \wedge t}\right) \\
= & F\left(\left\{A_{T_{n-1} \wedge t}+\left(X_{T_{n} \wedge t}-X_{T_{n-1} \wedge t}\right)-\int_{T_{n-1} \wedge t}^{T_{n} \wedge t} \int_{S} f(s, y, \omega) n\left(x_{s}, d y\right) d \varphi_{s}^{0}+\left(\varphi_{T_{n} \wedge t}-\varphi_{T_{n-1} \wedge t}\right)\right\}\right. \\
& -F\left(A_{T_{n-1} \wedge t}\right) \\
= & \int_{T_{n-1} \wedge t}^{T_{n} \wedge t} F^{\prime}\left(A_{s}\right) d X_{s}+\int_{T_{n-1} \wedge t}^{T_{n} \wedge t} F^{\prime}(A s) d \varphi_{s}+\int_{T_{n-1} \wedge t}^{T_{n} \wedge t} \int_{S} F^{\prime}\left(A_{s}\right) f(s, y, \cdot) n\left(x_{s}, d y\right) d \varphi_{s}^{0} \\
& +\frac{1}{2} \int_{T_{n-1} \wedge t}^{T_{n} \wedge t} F^{\prime \prime}\left(A_{s}\right) d<X>_{s} .
\end{aligned}
$$

On the other hand,

$$
\begin{aligned}
& \sum\left\{F\left(A_{T_{n} \wedge t}\right)-F\left(F_{T_{n} \wedge t^{-}}\right)\right\} \\
& =\int_{0}^{t} \int_{S}\left\{F\left(A_{s}+f(s, y, \omega)\right)-F\left(A_{s}\right)\right\} P(d s, d y) \\
& +\int_{0}^{t} \int_{S}\left\{F\left(A_{s}+g(s, y, \omega)\right)-F\left(A_{s}\right)\right\} P(d s, s y) \\
& =\int_{0}^{t} \int_{S}\left\{F\left(A_{s}+f(s, y, \omega)\right)-F\left(A_{s}\right)\right\} q(d s, d y) \\
& +\int_{0}^{t} \int_{S}\left\{F\left(A_{s}+g(s, y, \omega)\right)-F\left(A_{s}\right)\right\} P(d s, d y) \\
& -\int_{0}^{t} \int_{S}\left\{F\left(A_{s}+f(s, y, \omega)\right)-F\left(A_{s}\right)\right\} n\left(x_{s}, d y\right) d \varphi_{s}^{0} .
\end{aligned}
$$

Substituting the above calculation to

$$
F\left(A_{t}\right)-F\left(A_{0}\right)=\sum\left\{F\left(A_{T_{n} \wedge t}\right)-F\left(A_{T_{n} \wedge t^{-}}\right)\right\}+\sum\left\{F\left(A_{T_{n} \wedge t^{-}}\right)-F\left(A_{T_{n-1} \wedge t}\right)\right\},
$$

we get the desired formula. 
Now let us consider the general case. Without loss of generality, we may assume that $X_{t}, Q_{f}$ are of $\mathfrak{M}$ and $\varphi_{t}$ is of $\mathfrak{A}_{c}$, i.e.,

$$
E .\left(\langle X\rangle_{t}\right)<\infty, E \cdot\left[\int_{0}^{t}\left\{\int f(s, y, \omega)^{2} n\left(x_{s}, d y\right)\right\} d \varphi_{s}^{0}\right]<\infty \text { and } E .\left(\varphi_{t}\right)<\infty .
$$

Let $I^{(i)} ; i=1, \ldots \ldots, 6$ be the 1 -st, ...., 6-th term of the right hand side of $(5.7)$, respectively. Set $f_{n}(s, y, \omega)=\chi_{\varepsilon_{n}}\left(x_{s}, y\right) \chi_{E_{n}}(y) f(s, y, \omega)$; let $\varepsilon_{n} \downarrow 0$ and $E_{n} \uparrow S$ $\left(E_{n} \in \Gamma_{\varepsilon_{n}}\right)$. Then $\left\|f-f_{n}\right\|_{Q}^{t, x} \rightarrow 0$ as $n \rightarrow \infty$, so that $Q_{f_{n}}(t)$ converges to $Q_{f}$ in \|\|$_{t, x}$-norms. We may assume that $Q_{f_{n}}(t)$ converges uniformly to $Q_{f}(t)$ on any compact set of $[0, \infty)$, because we can choose such subsequence from $\left\{Q_{f_{n}}\right\}$. Set $A_{t}^{n}=X_{t}+Q_{f_{n}}+\varphi_{t}+P_{g}(t)$. Then (5.7) holds for $A_{t}=A_{t}^{n}$. Let $I_{n}^{(i)} ; n=1, \ldots \ldots$, $i=1, \ldots \ldots, 6$ be the corresponding terms to $I^{(i)}$ when $A_{t}$ is replaced by $A_{t}^{n}$. Since $\left\{I_{n}^{(i)}\right\}, i=3, \ldots \ldots, 6$ are Stieltjes integrals for almost all $\omega$, it is clear that $I_{n}^{(i)}$ converge to $I^{(i)}$. We shall show that $I_{n}^{(1)} \rightarrow I^{(1)}$ and $I_{n}^{(2)} \rightarrow I^{(2)}$ if (i) is satisfied. Since $A_{s}^{n}$ converges to $A_{s}$ uniformly on $[0, t)$,

$$
E .\left(\left|I^{(1)}-I_{n}^{(1)}\right|^{2}\right)=E \cdot\left[\int_{0}^{t}\left\{F^{\prime}\left(A_{s}\right)-F^{\prime}\left(A_{s}^{n}\right)\right\}^{2} d<X>_{s}\right] \rightarrow 0 \text { as } n \rightarrow \infty \text {. }
$$

Therefore $\lim I_{n}^{(1)}=I^{(1)}$. Now, by the mean value theorem

$$
\begin{gathered}
F\left(A_{s}+f(s, y, \cdot)\right)-F\left(A_{s}\right)=F^{\prime}\left(A_{s}+\tilde{f}(s, y, \cdot)\right) f(s, y, \cdot), \\
F\left(A_{s}^{n}+f_{n}(s, y, \cdot)\right)-F\left(A_{s}^{n}\right)=\chi_{\varepsilon n}\left(x_{s}, y\right) \chi_{E_{n}}(y) F^{\prime}\left(A_{s}^{n}+\tilde{f}_{n}(s, y, \cdot)\right) f(s, y, \cdot),
\end{gathered}
$$

where $\tilde{f}$ and $\tilde{f}_{n}$ are $(s, y, \omega)$-measurable functions satisfying $|\tilde{f}| \leq|f|$ and $\left|\tilde{f}_{n}\right| \leq\left|f_{n}\right|$.

Therefore

$$
\begin{aligned}
& E .\left(\left|I^{(2)}-I_{n}^{(2)}\right|^{2}\right) \\
& \leqq 2 E \cdot\left[\int_{0}^{t} \int_{s}\left\{\left(1-\chi_{\varepsilon_{n}}\left(x_{s}, y\right) \chi_{E_{n}}(y)\right) F^{\prime}\left(A_{s}^{n}+\tilde{f}_{n}(s, y, \cdot)\right)^{2} f(s, y, \cdot)^{2}\right\} n\left(x_{s}, d y\right) d \varphi_{s}^{0}\right] \\
& +2 E \cdot\left[\int _ { 0 } ^ { t } \int _ { S } \left\{\left(F^{\prime}\left(A_{s}^{n}+\tilde{f}_{n}(s, y, \cdot)-F\left(A_{s}+\tilde{f}(s, y, \cdot)\right)^{2} f(s, y, \cdot)^{2}\right\} n\left(x_{s}, d y\right) d \varphi_{s}^{0}\right] .\right.\right.
\end{aligned}
$$

Note that $F^{\prime}\left(A_{s}^{n}+\tilde{f}_{n}\right) \rightarrow F^{\prime}\left(A_{s}+\tilde{f}\right)$. Then each term of the right hand of the above converges to 0 . Therefore $I_{n}^{(2)} \rightarrow I^{(2)}$. Thus we have proved the theorem when $F^{\prime}$ is bounded. Now, suppose that (ii) is satisfied. Let $F_{M}$ be a $C^{2}$-class function such that $F_{M}^{\prime}$ is bounded and $F(x)=F_{M}(x)$ for $|x|<M+\sup |f|$. Then (5. 7) is satisfied for such $F_{M}$. Set $T_{M}=\inf \left\{t>0 ;\left|A_{t}\right|>M\right\}$. Clearly each term of (5.7) corresponding to $F_{M}$ is equal to each term corresponding to $F$ respectively for $t<T_{M}$. Thus (5.7) is satisfied for $F$. 


\section{\$ 6. A factorization of multiplicative functional.}

In this section, each functional $X_{t}(\omega)$ is assumed to be defined on $t \in[0, \zeta(\omega))$, i.e., it is right continuous and has left hand limits for $t \in[0, \zeta(\omega))$ and $\mathfrak{F}_{t}[\zeta>t]-$ measurable, where $\mathfrak{F}_{t}[\zeta>t]$ is the $\sigma$-field obtained by the restriction of $\mathfrak{F}_{t}$ on the set $\{\zeta>t\}$.

A nonnegative functional $\alpha_{t}(\omega)$ is called a multiplicative functional (m.f.), if $\alpha_{t}(\omega) \alpha_{s}\left(\theta_{t} \omega\right)=\alpha_{t+s}(\omega)$ holds for every $t, s>0$ such that $t+s<\zeta(\omega)$ and if $E_{x}\left(\alpha_{t}\right.$; $\zeta>t) \leqq 1$ is satisfied for every $x \in S$ and $t>0$. Ito, S. Watanabe [5] proved that any m.f. $\alpha_{t}$ can be factorized to the products of two m.f.'s such as $\alpha_{t}=\alpha_{t}^{(0)} \alpha_{t}^{(1)}$; where $\alpha_{t}^{(0)}$ is a local martingale and $\alpha_{t}^{(1)}$ is a natural decresing process, i.e., $\alpha_{t}^{(1)}(\omega)$ is a decresing function of $t$ and has jumps only at the continuity points of sample paths. The purpose of this section is to investigate the detailed structure of $\alpha_{t}^{(0)}$. Throughout this section, we will assume that the terminal time $\zeta$ is accessible, i.e., there exists a sequence of stopping times $T_{1} \leqq T_{2} \leq \ldots \ldots<\zeta$ converging to $\zeta$.

Theorem 6. 1. Let $\alpha_{t}$ be a m.f. satisfying $0<\alpha_{t}<\infty(t<\zeta)$. Then $\alpha_{t}$ is a local martingale if and only if; (i) there exists a $X_{t}^{c} \in \mathfrak{M}_{c}^{l o c} \cap \boldsymbol{A}$, (ii) there exists $B_{S \times S^{-}}$measurable function $f(x, y)$ satisfying $\int_{0}^{t} f_{1}^{2} \circ n\left(x_{s}\right) d \varphi_{s}^{0}<\infty(t<\zeta)$ and $\left|\int_{0}^{t}\left(e^{f_{2}}-1\right) \circ n\left(x_{s}\right) d \varphi_{s}^{0}\right|<\infty(t<\zeta)$, where $f_{1}(x, y)=f(x, y) \chi_{\{|f(x, y)|<1\}}$ and $f_{2}(x, y)=$ $f(x, y)-f_{1}(x, y)$, and (ii) $\varphi_{t}=\log \alpha_{t}$ is represented as

$$
\left.\varphi_{t}=X_{t}^{c}+Q_{f_{1}}(t)+P_{f_{2}}-\int_{0}^{t}\left(e^{f_{1}}-1-f_{1}\right) \circ n\left(x_{s}\right) d \varphi_{s}^{0}-\int_{0}^{t}\left(e^{f_{2}}-1\right) \circ n\left(x_{s}\right) d \varphi_{s}^{0}-\frac{1}{2}\left\langle X^{c}\right\rangle_{t}{ }^{8}\right)
$$

Lemma 6.1. Let $X_{t}^{c} \in \mathfrak{M}_{c}^{l o c} \cap \boldsymbol{A}, Q_{f} \in \mathfrak{M}_{d}^{l o c} \cap(f ;$ bounded $), \varphi_{t} \in \mathfrak{A}_{c}^{\text {loc }} \cap \boldsymbol{A}$ and $P_{g}(t)$ a purely discontinuous q.1.c. a.f. such that $g f=0$. Then

$$
\alpha_{t}=\exp \left(X_{t}^{c}+Q_{f}(t)+\varphi_{t}+P_{g}(t)\right)
$$

is a local martingale if and only if

$$
\int_{0}^{t}\left(e^{g}-1\right) \circ\left(n x_{s}\right) d \varphi_{s}^{0}<\infty \quad \text { for } t<\zeta .
$$

8) $\int_{0}^{t} f^{\circ} n\left(x_{s}\right) d \varphi_{s}^{0}=\int_{0}^{t}\left\{\int_{s} f\left(x_{s}, y\right) n\left(x_{s}, d y\right)\right\} d \varphi_{s}^{0}$.

It should be remarked that in the sequel the notation $Q_{h}(t)=\int_{0}^{t} h\left(x_{s}, y\right) q(d s, d y)$ for $h=h(x, y)$ is understood as $Q_{h^{\prime}}(t)$ of $\S 5$ for $h^{\prime}(t, y, \omega)=h\left(x_{t-}(\omega), y\right)$. So this notation is the same as in [11]. The same remark should be applied for $P_{h}(t)=\int_{0}^{t} h\left(x_{s}, y\right) P(d s, d y)$. 


$$
-\varphi_{t}=\int_{0}^{t}\left\{\left(e^{f}-1-f\right) \circ n\left(x_{s}\right)+\left(e^{g}-1\right) \circ n\left(x_{s}\right)\right\} d \varphi_{s}^{0}+\frac{1}{2}\left\langle X^{c}\right\rangle_{t} \text { for } t<\zeta
$$

Proof. 1. By the formula on stochastic integral (5. 7), we have

$$
\begin{aligned}
\alpha_{t}-1 & =\int_{0}^{t} \alpha_{s} d X_{s}+\int_{0}^{t} \int_{S}\left(e^{f\left(x_{s}, y\right)}-1\right) q(d s, d y)+\int_{0}^{t} \alpha_{s} d \varphi_{s}+\frac{1}{2} \int_{0}^{t} \alpha_{s} d\left\langle X^{c}\right\rangle_{s} \\
& +\int_{0}^{t} \alpha_{s}\left(e^{f}-1-f\right) \circ n\left(x_{s}\right) d \varphi_{s}^{0}+\int_{0}^{t} \int_{S} \alpha_{s}\left(e^{g\left(x_{s}, y\right)}-1\right) P(d s, d y) .
\end{aligned}
$$

If (1) is satisfied, $\int_{0}^{t} \alpha_{s}\left(e^{g}-1\right) \circ n\left(x_{s}\right) d \varphi_{s}<\infty, \quad$ so that $\int_{0}^{t} \alpha_{s}\left(e^{g}-1\right) P(d s, d y)$ $-\int_{0}^{t} \alpha_{s}\left(e^{g}-1\right) \circ n\left(x_{s}\right) d \varphi_{s}^{0}$ is a local martingale. Hence under (1),

$$
\begin{aligned}
\int_{0}^{t} \alpha_{s} d \varphi_{s}+1 / 2 \int_{0}^{t} \alpha_{s} d\left\langle X^{c}\right\rangle_{s} & +\int_{0}^{t} \alpha_{s}\left(e^{f}-1-f\right) \circ n\left(x_{s}\right) d \varphi_{s}^{0} \\
& +\int_{0}^{t} \alpha_{s}\left(e^{g}-1\right) \circ n\left(x_{s}\right) d \varphi_{s}^{0}=0
\end{aligned}
$$

is a necessary and sufficient condition that $\alpha_{t}-1$ is a local martingale (see Corollary of Theorem 1.3). It is clear that (6.3) is equivalent to (2). Therefore we have only to prove (1) if $\alpha_{t}$ is a local martingale.

2. Set $g^{+}=g \vee 0$ and $g^{-}=g \wedge 0$. We shall prove first that

$$
\left|\int_{0}^{t}\left(e^{g-}-1\right) \circ n\left(x_{s}\right) d \varphi_{s}^{0}\right|<\infty \text { for } t<\zeta
$$

From (6. 2),

$$
e^{P_{q}-(t)}-1=-\int_{0}^{t}\left(1-e^{g-\left(x_{s-}, y\right)}\right) e^{P_{q}-(s)} P(d s, d y)
$$

Therefore

$$
E \cdot\left(e^{P_{q}-(t)}-1\right)=-E \cdot\left(\int_{0}^{t}\left\{\int\left(1-e^{g-\left(x_{s}, y\right)}\right) e^{P_{g}-(s)} n\left(x_{s}, d y\right)\right\} d \varphi_{s}^{0}\right) .
$$

which shows that the right hand of (6.5) is finite, implying (6.4).

Next we shall show $\int_{0}^{t}\left(e^{g^{+}}-1\right) \circ n\left(x_{s}\right) d \varphi_{s}<\infty(t<\zeta)$. Let $\left\{g_{n}^{+}(x, y)\right\}$ be a nonnegative increasing sequence of bounded $B_{S \times S^{-}}$-measurable function such that $g_{n}^{+} \uparrow g^{+}$and $Q_{g_{+}} \in \mathfrak{M}_{d}^{l o c} \cap A$. Set $\alpha_{t}^{n}=\alpha_{t} \exp \left(P_{g_{n}^{+}}-P_{g^{+}}\right)$. Then we get $\alpha_{t}^{n}-1=$ local martingale $+\int_{0}^{t} \alpha_{s}^{n} d \varphi_{s}+\frac{1}{2} \int_{0}^{t} \alpha_{s}^{n} d\left\langle X^{c}\right\rangle_{s}-\int_{0}^{t}\left(e^{g_{n}^{+}}-1\right) \circ n\left(x_{s}\right) d \varphi_{s}^{0}$. 
Let $\left\{T_{m}\right\}$ be an increasing sequence of stopping times converging to $\zeta$ and satisfying that the local martingale part, $\int_{0}^{t \wedge T_{m}} \alpha_{s} d \varphi_{s}$ and $\int_{0}^{t \wedge T_{m}} \alpha_{s} d\left\langle X^{c}\right\rangle_{s}$ are all integrable. Then we have

$$
\begin{aligned}
E .\left(\alpha_{t \wedge T_{m}}^{n}\right)-1 & =E \cdot\left(\int_{0}^{t \wedge T_{m}} \alpha_{s}^{n} d \varphi_{s}\right)+\frac{1}{2} E \cdot\left(\int_{0}^{t \wedge T_{m}} \alpha_{s}^{n} d\left\langle X^{c}\right\rangle_{s}\right) \\
& -E \cdot\left(\int_{0}^{t \wedge T_{m}} \alpha_{s}^{n}\left(e^{g_{n}^{+}}-1\right)_{n}^{\circ}\left(x_{s}\right) d \varphi_{s}^{0}\right) .
\end{aligned}
$$

Noting that $\alpha_{t}^{n}$ increases to $\alpha_{t}$, we get

$$
\begin{aligned}
E .\left(\alpha_{t \wedge T_{m}}\right)-1 & =E .\left(\int_{0}^{t \wedge T_{m}} \alpha_{s} d \varphi_{s}\right)+\frac{1}{2} E .\left(\int_{0}^{t \wedge T_{m}} \alpha_{s} d\left\langle X^{c}\right\rangle_{s}\right. \\
& -E .\left(\int_{0}^{t \wedge T_{m}} \alpha_{s}\left(e^{g^{+}}-1\right) \circ n\left(x_{s}\right) d \varphi_{s}^{0}\right),
\end{aligned}
$$

which shows that the last term of the right hand is finite. Thus $\int_{0}^{t}\left(e^{g^{+}}-1\right) \circ n\left(x_{s}\right) d \varphi_{s}^{0}$ is finite. Since $\left|e^{g}-1 \leqq\right| e^{g^{+}}-1|v| e^{g^{-}}-1 \mid$, we obtain (1),

Proof of theorem 6.1. $1^{\circ}$. The sufficient condition has already been proved at Lemma 6. 1. To prove the necessary condition, we will show first that $\varphi_{t}$ is a q.1.c. a.f. Indeed, since $\alpha_{t \wedge T_{n}}$ is martingale, the discontinuous points of $\alpha_{t \wedge T_{n}}$ are the discontinuous points of sample paths. Thus $\varphi_{t}$ is a q.1.c. a.f. Set $\psi_{t}=\sum\left|\Delta \varphi_{s}\right| \geq 1, s \leq t<s \Delta \varphi_{s}$. Then $\psi_{t}$ can be written as $P_{g}(t)$ and $g$ satisfies $g(x, y) \chi_{\{|g(x, y)|<1\}}=0$ (see [11]). By the argument at the proof of Lemma 6.1, $\int_{0}^{t}\left(1-e^{g-}\right) \circ n\left(x_{s}\right) d \varphi_{s}^{0}$ is finite. Thus

$$
\beta_{t} \equiv \exp \left(P_{g-}(t)-\int_{0}^{t}\left(1-e^{g^{-}}\right) \circ n\left(x_{s}\right) d \varphi_{s}^{0}\right)
$$

is a local martingale by Lemma 6.1 .

$2^{\circ}$. We shall show that $\alpha_{t} \beta_{t}^{-1}$ is also a local martingale. By Lemma 6. 1, we get

$$
\beta_{t}^{-1}-1=\int_{0}^{t} \beta_{s}^{-1}\left(e^{\left.-g^{-\left(x_{s}\right.}, y\right)}-1\right) P(d s, d y)-\int_{0}^{t} \beta_{s}^{-1}\left(1-e^{g^{-}}\right) \circ n\left(x_{s}\right) d \varphi_{s}^{0}
$$

Let $\left\{T_{m}\right\}$ be an increasing sequence of stopping times such that $T_{m}<\zeta$, $T_{m} \uparrow \zeta, E_{x}\left(\int_{0}^{t \wedge T_{m}} \alpha_{s} \beta_{s}^{-1}\left(1-e^{g^{-}}\right) \circ n\left(x_{s}\right) d \varphi_{s}^{0}\right)<\infty \quad(t>0, x \in S) \quad$ and $\quad \alpha_{t \wedge T_{m}} \quad$ is a martingale. Then, for $B \in \mathfrak{F}_{s} \wedge T_{m}$ 


$$
\begin{aligned}
& E .\left(\alpha_{t \wedge T_{m}} \beta_{t}^{-1} \wedge_{m} ; B\right)-E .\left(\alpha_{s} \wedge T_{m} \beta_{s}^{-1} \wedge_{T_{m}} ; B\right) \\
& =E \cdot\left(\alpha_{t \wedge T_{m}} \int_{s \wedge T_{m}}^{t \wedge T_{m}} \int_{S}\left(e^{-g^{-}}-1\right) P(d s, d y) ; B\right)-E \cdot\left(\alpha_{t \wedge T_{m}} \int_{s \wedge T_{m}}^{t \wedge T_{m}} \beta_{s}^{-1}\left(1-e^{g^{-}}\right) \circ n\left(x_{s}\right) d \varphi_{s}^{0} ; B\right) \\
& =0 \text {. }
\end{aligned}
$$

$3^{\circ}$. Set $\gamma_{t}=\alpha_{t} \beta_{t}^{-1} \exp \left(-P_{g}+(t)\right)$ and $\psi_{t}=\log \gamma_{t}$. Then $\psi_{t}$ is a q.1.c. a.f. whose absolute values of jumps are less than 1 . Therefore $\psi_{t}$ is locally square integrable (i.e. $\exists_{n} \uparrow_{\infty} E$. $\left[\left|\psi_{T_{n}}\right|^{2}\right]<\infty$ ). Since $\log x$ is concave and increasing function and since $\gamma_{t}$ is a supermartingale, $\phi_{t}$ is a regular supermartingale belonging locally to the class $(D)$ (in the sense $T_{n} \uparrow \infty^{\prime} \psi_{t}^{(n)} \equiv \psi_{t} \wedge T_{n}$ is class $(D)$ ) by Jensen's inequality. Therefore, by Meyer's decomposition, there exists a unique $A_{t}$ of $\mathfrak{A}_{c}^{+l 0 c}$ such that $X_{t} \equiv \psi_{t}+A_{t}$ is a local martingale. Moreover, we can prove that $A_{t}$ is an a.f. ${ }^{9)}$ Furthermore, $X_{t}$ can be decomposed to sums of $X_{t}^{c} \in \mathfrak{M}_{c}^{l o c} \cap \boldsymbol{A}$ and $Q_{h} \in \mathfrak{M}_{d}^{l o c} \cap \boldsymbol{A} .^{10)} \quad$ Thus we have proved that

$$
\varphi_{t}=X_{t}^{c}+Q_{h}(t)+A_{t}+P_{g+}(t)+P_{g-}(t)-\int_{0}^{t}\left(e^{g^{-}}-1\right) \circ n\left(x_{s}\right) d \varphi_{s}^{0} .
$$

Since $Q_{h}$ and $P_{g}$ have no common jumps, $g . h=0$. Define $f(x, y)$ by $g(x, y)$ if $|g(x, y)| \geq 1$ and $h(x, y)$ if $0<|h(x, y)|<1$. Then $\varphi_{t}$ can be written as

$$
\varphi_{t}=X_{t}^{c}+Q_{f_{1}}(t)+A_{t}+P_{f_{2}}(t)-\int_{0}^{t}\left(e^{f_{2}}-1\right) \circ n\left(x_{s}\right) d \varphi_{s}^{0}
$$

Applying Lemma 6. 1, we get the desired formula.

From Theorem 6. 1, a m.f. which is a local martingale, can be factorized to the product of two m.f.'s $\beta_{t}$ and $\gamma_{t} ; \beta_{t}$ is a continuous m.f. represented as $\exp \left(X_{t}^{c}-\frac{1}{2}\left\langle X^{c}\right\rangle_{t}\right)$ and $\gamma_{t}$ is a discontinuous m.f. represented as $\exp \left(Q_{f_{1}}(t)+P_{f_{2}}(t)-\int_{0}^{t}\left(e^{f_{1}}-1-f_{1}\right) \circ n\left(x_{s}\right) d \varphi_{s}^{0}-\int_{0}^{t}\left(e^{f_{2}}-1\right) \circ n\left(x_{s}\right) d \varphi_{s}^{0}\right), \quad$ both of which are local martingales. Further, $\beta_{t}$ and $\gamma_{t}$ are orthogonal in the sense that the product of two local martingales is also a local martingale. It should be noted that the discontinuous part $\gamma_{t}$ is uniquely determined by $B_{S \times S}$-measurable function $f(x, y)$. We will call $\gamma_{t}$ to be generated by $f(x, y)$.

The situation of jumps of sample paths is closely related to Lévy system $\left(n(x, d y), \varphi_{t}^{0}\right)$. It is interesting to know how the Lévy system may be changed by the transformation of Markov process by a m.f. $\alpha_{t}$. The change of Lévy

9) It is not clear that $A_{t}$ is an a.f. from Meyer's decomposition. We were informed the proof from Tanaka by private comunication ommitting here.

10) If $X_{t}$ is an a.f., then $X_{t}^{c}$ and $Q_{f}$ are also a.f.'s. See [10]. 
system depends only on discontinuous part of $\alpha_{t}$. Precisely,

Theorem 6. 2. Let a m.f. $\alpha_{t}\left(0<\alpha_{t}<\infty\right.$ for $\left.t<\zeta\right)$ be a local martingale and let it be represented as Theorem 6.1. Let $M^{\alpha}=\left(X_{t}^{\alpha}, \zeta^{\alpha}, F_{t}^{\alpha}, P_{x}^{\alpha}\right)^{11)}$ by a standard process which is obtained from transformation by $\alpha_{t}$. Then $\left(e^{f(x, y)} n(x, d y), \varphi_{t}^{0}\right)$ becomes a Levy system of $M^{\alpha}$.

Proof. Let $h(x, y) \geqq 0$ be a $B_{S \times S}$-measurable function such that $E_{x}\left(P_{h}(t)^{2}\right)$ $<\infty$. Let $T<\zeta$ be a stopping time such that $\int_{0}^{t \wedge T} \alpha_{s} d X_{s}^{c} \in \mathfrak{M}_{c}, \alpha_{t} \wedge_{T}$ is a martingale and $E_{x}\left(\alpha_{t \wedge T} \int_{0}^{t \wedge T} h \circ n\left(x_{s}\right) d \varphi_{s}^{0}\right)<\infty$. Since

$$
\alpha_{t \wedge T}-1=\int_{0}^{t \wedge T} \int_{S} \alpha_{s}\left(e^{f(x s, y)}-1\right) q(d s, d y)+\int_{0}^{t \wedge T} \alpha_{s} d X_{s}^{c}
$$

we get

$$
\begin{aligned}
E_{x}\left(Q_{h}(t \wedge T) \alpha_{t \wedge T}\right) & =E_{x}\left(Q_{h}(t \wedge T) \int_{0}^{t \wedge T} \int_{S} \alpha_{s}\left(e^{f\left(x_{s}, y\right)}-1\right) q(d s, d y)\right) \\
& =E_{x}\left(\int_{0}^{t \wedge T} \alpha_{s}\left(e^{f}-1\right) h \circ n\left(x_{s}\right) d \varphi_{s}^{0}\right) .
\end{aligned}
$$

Thus we have

$$
\begin{aligned}
& E_{x}\left(\alpha_{t \wedge T} P_{h}(t \wedge T)\right)=E_{x}\left(\alpha_{t} \wedge T \int_{0}^{t \wedge T} h \circ n\left(x_{s}\right) d \varphi_{s}^{0}\right)+E_{x}\left(\int_{0}^{t \wedge T} \alpha_{s}\left(e^{f}-1\right) h \circ n\left(x_{s}\right) d \varphi_{s}^{0}\right) \\
& =E_{x}\left(\alpha_{t \wedge T} \int_{0}^{t \wedge T} e^{f} h \circ n\left(x_{s}\right) d \varphi_{s}^{0}\right),
\end{aligned}
$$

which implies

$$
\left.E_{x}^{\alpha}\left(P_{h}(t \wedge T)\right)=E_{x}^{\alpha}\left(\int_{0}^{t \wedge T} e^{f} h \circ n\left(x_{s}\right) d \varphi_{s}^{0}\right) .12\right)
$$

The above formula shows that $\left(e^{f(x, y)} n(x, d y), \varphi_{t}^{0}\right)$ is a Lévy system of $M^{\alpha}$ by the definition.

Example 1. Let $S$ be a countable set with discrete topology and $M=\left(x_{t}, \zeta, \mathfrak{F}_{t}, P_{x}\right)$ a markov chain (Hunt process) over $S$. Set $\tau_{1}(\omega)=\inf \{t>0$; $\left.x_{t}(\omega) \neq x_{0}(\omega)\right\}, q_{x}^{-1}=E_{x}\left(\tau_{1}\right), \quad \pi(x, y)=P_{x}\left(x_{\tau_{1}}=y\right) \quad$ and $\quad q_{x, y}=q_{x} \Pi(x, y)$. We will assume $0<q_{x}<\infty$ and $\sum_{y \in s} \pi(x, y)=1$. Set $P_{t}(x, y)=P_{x}\left(x_{t}=y\right)$. Since

$$
\lim _{t \rightarrow 0} \frac{P_{t}(x, y)}{t}=q_{x, y}(x \neq y) \text { and } \lim _{t \rightarrow 0} \frac{1-P_{t}(x, x)}{t}=q_{x},
$$

11) For the definition of $M^{\alpha}$, wee [6].

12) If $B$ is an element of $\mathfrak{P}_{t}$, then $P_{x}\left(\alpha_{t} ; B, \zeta>t\right)=P_{x}^{\alpha}(B ; \zeta>t)$. See [6]. 
$\left\{q_{x, y}, t\right\}$ is a Lévy system of $M$ (See S. Watanabe [11]) and $M$ is uniquely determined by $q_{x}$ and $q_{x, y}$, which means that there is no continuous part for any m.f. and every m.f. is generated by $S \times S$-function $f(x, y)$.

Let $f(x, y)$ be a finite valued function satisfying $\sum_{y \in S} e^{f(x, y)} \Pi(x, y)<\infty$. Then $\sum_{y \in S}\left(e^{f_{2}(x, y)}-1\right) q_{x, y}<\infty$ and $\sum_{y \in S} f_{1}(x, y)^{2} q_{x, y}<\infty ; \quad$ so that the m.f. $\alpha_{t}$ generated by such $f(x, y)$ is a local martingale. The process transformed by $\alpha_{t}$ has $\left\{e^{f(x, y)} q_{x, y}, t\right\}$ as its Lévy system. Conversely, let $M$ and $M^{\prime}$ be two Markov chains defined on the same state space $S$. The corresponding quantities relative to $M^{\prime}$ are denoted by $q_{x}^{\prime}, q_{x, y}^{\prime}$ and $\Pi^{\prime}(x, y)$. We will assume that $0<q_{x}<\infty, \quad 0<q_{x}^{\prime}<\infty$, and $\sum_{y \in S} \Pi(x, y)=\sum_{y \in S} \Pi^{\prime}(x, y)=1$. Assume further $q_{x, y}^{\prime}>0$ implies $q_{x, y}>0$. Define $f(x, y)=\log q_{x, y}^{\prime}-\log q_{x, y} . \quad$ It is easy to see that this $f(x, y)$ satisfies the condition (2) of Lemma 6.1. Therefore there exists a m.f. generated by $f(x, y)$. Since the process transformed by $\alpha_{t}$ has the Lévy system $\left\{e^{f(x, y)} q_{x, y}, t\right\}, M$ coincides with $M^{\prime}$. Thus we get the following statement: Let $M$ and $M^{\prime}$ be Markov chains defined on the same state space $S$ and let $0<q_{x}<\infty, 0<q_{x}^{\prime}<\infty$ and $\sum_{y \in S} \Pi(x, y)=\sum_{y \in S} \Pi^{\prime}(x, y)=1 . \quad A$ necessary and sufficient condition that $M^{\prime}$ can be obtained from transformations by a m. $f$. which is a local martingale is that $\Pi(x, y)=0$ implies $\Pi^{\prime}(x, y)=0$ and vice versa.

From the above argument, in the case of Markov chain, transformations by $m$. $f . ' s$ contains time changes by inverse functions of strictly increasing $a . f .^{\prime} s$ as its special case. Indeed, if a Markov chain $M$ has the system $\left\{q_{x}, \Pi(x, y)\right\}$, the time changed process $M^{\prime}$ has the same quantity $\Pi(x, y)$ and different quantity $q_{x}^{\prime}$; so that $f(x, y)=\log q_{x, y}^{\prime}-\log q_{x, y}=\log q_{x}^{\prime}-\log q_{x}$ is only a function of $x$, and the chain transformed by the $m$. $f$. generated by this $f(x)=f(x, y)$ coincides with the time changed process $M^{\prime}$.

Another special case is the case that $f(x, y)$ satisfies $\sum_{y \in S} e^{f(x, y)} \Pi(x, y)=1$. In this case, the transformed process has the same quantity $q_{x}$ as the original one. In particular, if $e^{f(x, y)}$ is of the form $h(x) / h(y)$, then $h(x)$ is a harmonic function and the transformation is nothing but (super)harmonic transformation (or $h$-path process).

ExAmple 2. Let $x_{t}=\left(B_{t}^{(1)}, \ldots \ldots, B_{t}^{(N)}\right)$ be an $N$-dimensional Brownian motion. Then every $X \in \mathfrak{M}_{c}^{l o c} \cap \boldsymbol{A}$ is represented as $X_{t}=\sum_{i=1}^{N} \int_{0}^{t} f^{(i)}\left(x_{s}\right) d B_{s}^{(i)}$, where $f^{(i)}, i=1, \ldots \ldots, N$ are measurable functions on $R^{N}$; so that $\langle X\rangle_{t}=\sum_{i=1}^{N} \int_{0}^{t} f^{(i)}\left(x_{s}\right)^{2} d s$. Therefore, every multiplicative functional being a local martingale can be 
represented as

$$
\exp \left\{\sum_{i=1}^{N} \int f^{(i)}\left(x_{s}\right) d B_{s}^{(i)}-\frac{1}{2} \sum_{i=1}^{N} \int f^{(i)}\left(x_{s}\right)^{2} d s\right\}
$$

This was obtained by Wentzel [12]. Transformation by such m.f. is so called that of "drift" and does not contain time change. This fact is quite different from the case of Markov chain in Example 1.

\section{§ 7. Decomposition of additive process.}

It is known (Lévy [7], Itô [4]) that any right continuous additive process may be decomposed uniquely to sum of a Gaussian additive process and a jump type additive process such that these are mutually independent. We shall prove this fact using the formula on stochastic integral obtained in $\$ 5$.

A right continuous stochastic process $\left(X_{t}, P\right), t \in T=[0, b]$ is called an additive process (or process with independent increments) if, $X_{t_{i}}-X_{t_{i-1}}, i=1,2$, $\ldots \ldots, n$, are independent if $0 \leqq t_{0}<t_{1}<t_{2}<\ldots \ldots<t_{n} \leqq b$. For the simplicity we shall assume that $X_{t}$ has no fixed discontinuity and $X_{0}=0$ a.e. $P$. Set

$$
P_{t}(E)=\sum_{\substack{s \leq t \\ \mid X_{s}-\bar{X}_{s-1}>0}} x_{E}\left(X_{s}-X_{s-}\right) \text { and } \sigma(t, E)=E\left(P_{t}(E)\right)
$$

It is easy to see that $P_{t}(E)$ is an additive process. Define $X_{t}^{1}=\int_{|X|>1} x P_{t}(d x)$. Then $X_{t}-X_{t}^{1}$ is an additive process whose absolute values of jumps are less than or equal to 1 , so that $X_{t}-X_{t}^{\mathbf{1}}$ is locally integrable. Define

$$
X_{t}^{2}=\lim _{\varepsilon \downarrow 0}\left\{\int_{\varepsilon<|X| \leq 1} x P_{t}(d x)-\int_{\varepsilon<|X| \leq 1} x \sigma(t, d x)\right\} .
$$

Then $X_{t}^{2}$ is a locally square integrable martingale and is an additive process. Set

$$
X_{i}^{j}=X_{t}^{1}+X_{t}^{2} \text { and } X_{t}^{c}=X_{t}^{j}-X_{t}^{j}
$$

Since $X_{t}^{c}$ is a continuous additive process, it is a Gaussian process. ${ }^{13)}$ Set $m(t)=E\left(X_{t}^{c}\right)$ and $X_{t}^{0}=X_{t}^{c}-m(t)$.

Theorem 7.1. The decompostion $X_{t}=X_{t}^{0}+X_{t}^{j}+m(t)$ satisfies the following conditions: (1)

$$
E\left(e^{i \alpha X_{t}^{0}}\right)=\exp \left\{-\frac{1}{2} \alpha^{2} v(t)\right\} \quad \text { where } \quad v(t)=E\left(\left|X_{t}^{0}\right|^{2}\right)
$$

13) See for example [4]. 


$$
E\left(e^{i \beta X_{t}^{j}}\right)=\exp \int_{|x| \leqq 1}\left(e^{i \beta x}-1-i \beta x\right) \sigma(t, d x)+\int_{|x|>1}\left(e^{i \beta x}-1\right) \sigma(t, d x) .
$$

(2) $X_{t}^{0}$ and $X_{t}^{j}$ are mutually independent.

Proof. To prove that $X_{t}^{0}$ and $X_{t}^{j}$ are mutually independent, it is sufficient to show that for every real $\alpha, \beta$,

$$
E\left(e^{i A\left(X_{t}^{0}-X_{s}^{0}\right)} e^{i \beta\left(X_{t}^{j}-X_{s}^{j}\right)}\right)=E\left(e^{i A\left(X_{t}^{0}-X_{s}^{0}\right)}\right) E\left(e^{i \beta\left(X_{t}^{j}-X_{s}^{j}\right)}\right) .
$$

Note that $X_{t}^{0}$ and $X_{t}^{j}$ are a.f.'s and local martingales and $X_{t}^{1}$ is a purely discontinuous q.1.c. a.f., relative to $\left(\tilde{x}_{t}^{(s, x)}, P\right)$. The argument at $\S 5$ together with Lemma 7.1 implies that $X_{t}^{j}$ is represented as $Q_{f}(t)+P_{f}(t)$, where $f((s, x),(t, y))=y-x . \quad$ Set $F\left(x^{1}, x^{2}\right)=e^{i\left(x^{1}+x^{2}\right)}, A_{t}^{(1)}=\alpha X_{t}^{0} \quad$ and $\quad A_{t}^{(2)}=\beta X_{t}^{j} \quad$ and apply Theorem $5.1,{ }^{14)}$ we have

$$
\begin{gathered}
\gamma_{s, t}^{\alpha, \beta}=\text { local martingale }+\int_{s}^{t} \gamma_{s, u}^{\alpha, \beta} \int_{\left|y-X_{u}\right| \leq 1}\left\{e^{i \beta\left(y-X_{u}\right)}-1-i \beta\left(y-X_{u}\right)\right\} \sigma\left(d u, d y-X_{u}\right) \\
+\int_{s}^{t} \gamma_{s, u}^{\alpha, \beta} \int_{\left|y-X_{u}\right|>1}\left(e^{i \beta\left(y-X_{u}\right)}-1\right) \sigma\left(d u, d y-X_{u}\right)-\frac{1}{2} \alpha^{2} \int_{s}^{t} \gamma_{s, u}^{\alpha, \beta} v(d u)
\end{gathered}
$$

where $\gamma_{s, t}^{\alpha, \beta}=e^{i \alpha\left(X_{t}^{0}-X_{s}^{0}\right)+i \beta\left(X_{t}^{j}-X_{s}^{j}\right)}$. Therefore,

$$
\begin{aligned}
& E\left(\gamma_{s, t}^{\alpha, \beta}\right) \\
& =\int_{s}^{t} E\left(\gamma_{s, u}^{\alpha, \beta}\right)\left\{-\frac{1}{2} \alpha^{2} v(d u)+\int_{|x| \leq 1}\left(e^{i \beta x}-1-i \beta x\right) \sigma(d u, d x)+\int_{|x|>1}\left(e^{i \beta x}-1\right) \sigma(d u, d x)\right\} .
\end{aligned}
$$

The above integral equation has a uniq : solution

$$
\begin{aligned}
& E\left(\gamma_{s, t}^{\alpha, \beta}\right)=\exp \left[-\frac{1}{2} \alpha^{2}\{v(t)-v(s)\}+\int_{|x| \leq 1}\left(e^{i \beta x}-1-i \beta x\right) \sigma((s, t], d x)\right. \\
& \left.+\int_{|x|>1}\left(e^{i \beta x}-1\right) \sigma((s, t], d x)\right] \\
& =E\left(\gamma_{s, t}^{\alpha, 0}\right) E\left(\begin{array}{c}
0, \beta \\
s, t
\end{array}\right) .
\end{aligned}
$$

Thus we have proved the theorem.

Remark. (1) When $X_{t}$ is temporally homogeneous, it is easy to see that $X_{t}^{0}$ and $X_{t}^{\jmath}$ are also temporally homogeneous and hence $X_{t}^{0}$ is a Brownian motion.

14) If $X_{t}$ is temporally homogenous, $X_{t}$ may be considered as a Hunt process and it is easy to see that $(n(x, E), t)$ becomes a Levy system, where $n(x, E)=\sigma(1, E-x)$. If $X_{t}$ is not temporally homogeneous, $X_{t}$ is no longer a Hunt process, obviously. However, $\sigma(t, E)$ works similarly as Levy system does in Hunt process. In fact $\sum_{s \leqq t} f\left(x_{s-}, x_{s}\right)-\int_{0}^{t} \int f\left(x_{u}, y\right) \sigma(d u, d y)$ is a martingale if it is integrable, where $\sigma((s, t, E)=\sigma(t, E)-\sigma(s, E)$. Thorem 5.1 is applicable with a slight modification. 
(2) In the same way as above we can prove easily $P_{t}(E)$ is a Poisson process such that if $E \cap F=\phi$ then $\left\{P_{t}(E)\right\}$ and $\left\{P_{t}(F)\right\}$ are mutually independent.

\section{§ 8. Appendix. Supermartingale and the corresponding increasing process of a Markov process.}

The material treated in this section is certain refinements and generalization of the results of $P$.Meyer [8, 9] in the case of Hunt process.

Let $M=\left(x_{t}, \zeta, \mathfrak{F}_{t}, P_{x}\right)$ be a Hunt process. An increasing process $A_{t}$ is said to belong to the class $(U)$ if $A_{t}$ has no common jumps with sample paths, i.e.

$$
P_{x}\left(A_{t} \neq A_{t-} \text { and } x_{t} \neq x_{t-} \text { for some } t>0\right)=0, \quad \forall x \in S .
$$

The set of all $A_{t}$ belonging to the class $(U)$ is denoted by $\mathfrak{A}_{U}$.

Theorem 8.1. Let $X_{t}$ be a supermartingale belonging to the class $(D)$. Suppose that $\lim _{t \rightarrow \infty} X_{t}=0$ holds a.e. $P_{x}$. There exists a unique $A_{t}$ of $\mathfrak{A}_{U}$ satisfying

$$
X_{t}=E_{x}\left(A_{\infty} / \mathfrak{F}_{t}\right)-A_{t} \quad \text { a.e. } \quad P_{x}, \quad \forall t>0, \quad \forall x \in S,
$$

where $A_{\infty}=\lim _{t \rightarrow \infty} A_{t} . \quad$ Furthermore, if $X_{t}$ is regular then $A_{t}$ is of $\mathfrak{A}_{c}^{+}$.

The above $A_{t}$ is said to generate $X_{t}$. The theorem is a modification of a fundamental result of [8.9]. It follows from [8,9] that for each $x$ there exists an increasing process $A_{t}^{(x)}$ such that $X_{t}=E\left(A_{\infty}^{(x)} / \mathfrak{F}_{t}\right)-A_{t}^{(x)}$ but it is not clear at the first sight that there exists a functional $A_{t}$ such that $A_{t}=A_{t}^{(x)}$ for each $x$.

Let $T$ be a stopping time. According to [9], $T$ is said to be totally inaccessible (in the weak sense), if it is strictly positive not infinite a.e. $P_{x}$ for some $x$ and for each increasing sequence $\left\{T_{n}\right\}$ of stopping times converging to $T$,

$$
P_{x}\left(\forall n, T_{n}<T<\infty\right)=0, \quad \forall x \in S .
$$

$T$ is said to be inaccessible if there exists $A$ of $\mathfrak{F}_{T}$ such that $P_{x}(A)>0$ for some $x$ and the stopping time

$$
\begin{aligned}
& T_{A}(\omega)=T(\omega) \quad \text { if } \quad \omega \in A \\
& =\infty \quad \text { if } \omega \in A
\end{aligned}
$$

is totally inaccessible. Further, $T$ is said to be accessible if $T$ is not inaccessible.

The following theorem is proved in [9] in the case that semi-group operator of the Hunt process maps $C(S)$ into $C(S) .{ }^{15)}$

15) $C(S)$ is the space of all bounded continuous functions on $S$. 
TheOREM 8. 2. Let $T$ be a stopping time. (1) $T$ is accessible if and only if

$$
P_{x}\left(x_{T}=x_{r-}, \quad T<\infty\right)=P_{x}(T<\infty), \forall x \in S,
$$

where $x_{T-}$ is the left-hand limit of sample paths $x_{t}$ at $T$, where $x_{0}=x_{0-}$.

(2) $T$ is totally inaccessible if and only if

$$
P_{x}\left(x_{T} \neq x_{T-}, \quad T<\infty\right)=P_{x}(T<\infty), \quad \forall x \in S .
$$

(3) There exist accessible stopping time $T_{1}$ and totally inaccessible stopping time $T_{2}$ satisfying $T=\min \left(T_{1}, T_{2}\right)$, and for any such $T_{1}$ and $T_{2}$

$$
\begin{aligned}
& \left.\left\{T_{1}=T<\infty\right\} \underset{a . s .}{=} \underset{x_{T}}{=} x_{T-}, T<\infty\right\} \\
& \left\{T_{2}=T<\infty\right\} \underset{\substack{a . s}}{=}\left\{x_{T} \neq x_{T-}, T<\infty\right\} .
\end{aligned}
$$

The stopping time defined by

$$
T_{1}= \begin{cases}T & \text { on }\left\{x_{T}=x_{T-}, T<\infty\right\} \\ +\infty & \text { otherwise }\end{cases}
$$

is called the accessible part of $T$.

Meyer [9] called an incresing process $A_{t}$ to be natural if, for any stopping time $T$ totally inaccessible, $P_{x}\left(A_{T} \neq A_{T^{-}}\right)=0$ holds $(\forall x \in S)$. From Theorem 8. 2, we have immediately

Corollary. An increasing process $A_{t}$ is natural if and only if it is of $\mathfrak{A}_{U}^{t}$.

Before the proof of these theorems, we prepare several lemmas.

Lemma 8. 1 Let $X$ be a regular supermartingale belonging to the class $(D)$ for each $P_{x}$. There exists $A_{t}$ of $\mathfrak{A}_{c}^{+}$satisfying (8.1).

Proof. $1^{\circ}$ Let $p_{h} X_{t}(h>0)$ be a right-continuous version of $E .\left(X_{t+h} / \mathfrak{F}_{t}\right)$. It is easy to see that $p_{h} X_{t}$ can be defined independently of the family of measures $P_{x}, x \in S$. Set

$$
A_{t}^{h}=\int_{0}^{t} \frac{1}{h}\left(X_{s}-p_{h} X_{s}\right) d s \text { and } X_{t}^{h}=E .\left(A_{\infty}^{h} / \mathfrak{F}_{t}\right)-A_{t}^{h} .
$$

We shall prove

$$
P_{x}\left(\sup _{t}\left|A_{t}^{h}-A_{t}^{k}\right|>\varepsilon\right) \rightarrow 0
$$

as $h$ and $k$ tend to 0 . It is known in [8] that, if $X_{t}$ is bounded, $E_{x}\left(\left|A_{\infty}^{h}-A_{\infty}^{k}\right|^{2}\right)$ converges to 0 as $h$ and $k$ tend to 0 , and that in general case, there exists a 
sequence of bounded regular supermartingales $X_{t}^{n}(x)$ relative to a fixed measure $P_{x}$ such that $X_{t}=\sum_{n=1}^{\infty} X_{t}^{n}(x)$ a.e. $P_{x}$. Set

$$
A_{t}^{n, k}(x)=\int_{0}^{t} \frac{1}{k}\left(X_{s}^{n}(x)-E x\left(X_{s+k}^{n}(x) / \Re_{s}\right) d s .\right.
$$

Then $E_{x}\left(\left|A_{t}^{n, h}(x)-A_{t}^{n, k}(x)\right|^{2}\right)$ converge to 0 as $h$ and $k$ tend to 0 . Since

$$
\begin{aligned}
& E_{x}\left(\left|A_{\infty}^{h}-A_{\infty}^{k}\right|\right) \leqq \sum_{n=1}^{\infty} E_{x}\left(\left|A_{\infty}^{n, h}(x)-A_{\infty}^{n, k}(x)\right|\right), \\
& E_{x}\left(\left|A_{\infty}^{n, h}(x)-A_{\infty}^{n, k}(x)\right|\right) \leqq 2 E_{x}\left(X_{0}^{n}(x)\right), \\
& \sum_{n=1}^{\infty} E_{x}\left(X_{0}^{n}(x)\right)=E_{x}\left(X_{0}\right)<\infty .
\end{aligned}
$$

The Lebesgue convergence theorem is applicable and we get $\lim _{h, k \rightarrow 0} E_{x}\left(\left|A_{\infty}^{h}-A_{\infty}^{k}\right|\right)$ $=0$. Therefore, by Doob's inequality concerning martingale, we get

$$
\varepsilon P_{x}\left(\sup _{t}\left|E_{x}\left(A_{\infty}^{h}-A_{\infty}^{k} \mid \mathfrak{F}_{t}\right)\right|>\varepsilon\right) \leqq E_{x}\left(\left|A_{\infty}^{h}-A_{\infty}^{k}\right|\right)
$$

On the otherhand

$$
\left|X_{t}^{h}-X_{t}^{k}\right| \leqq\left|X_{t}-X_{t}^{h}\right| \leqq X_{t}-p_{h} X_{t} \text { if } h>k,
$$

and we get by the argument of Shur-Meyer (e.g. [9]),

(8. 7) $P_{x}\left(\sup _{t}\left|X_{t}^{h}-X_{t}^{k}\right|>\varepsilon\right) \leqq P_{x}\left(\sup _{t}\left|X_{t}-X_{t}^{h}\right|>\varepsilon\right) \leqq P_{x}\left(T_{h}<\infty\right) \rightarrow 0,(h, k \rightarrow 0)$,

where $T_{h}=\inf \left\{t>0 ; X_{t}-p_{h} X_{t}>\varepsilon\right\}$. Consequently, from (8.6) and (8.7), we get

$$
\begin{aligned}
P_{x}\left(\sup _{t}\left|A_{t}^{h}-A_{t}^{k}\right|>\varepsilon\right) & \leqq P_{x}\left(\sup _{t}\left|E_{x}\left(A_{\infty}^{h}-A_{\infty}^{k} / \mathfrak{F}_{t}\right)\right|>\frac{\varepsilon}{2}\right) \\
& +P_{x}\left(\sup _{t}\left|X_{t}^{h}-X_{t}^{k}\right|>\frac{\varepsilon}{2}\right) \rightarrow 0, \quad(h, k \rightarrow 0) .
\end{aligned}
$$

$2^{\circ}$. To prove the lemma, it is sufficient to show that there exists $A_{t}$ of $\mathfrak{A}_{c}^{+}$ satisfying $P_{x}\left(\sup _{t}\left|A_{t}-A_{t}^{h}\right|>\frac{\varepsilon}{2}\right) \rightarrow 0$ as $h$ tends to 0 . Let $\left\{\varepsilon_{n}\right\}(>0)$ be a decreasing sequence satisfying $\sum \varepsilon_{n}<\infty$. Set $e_{n}(x, h, k)=P_{x}\left(\sup _{t}\left|A_{t}^{h}-A_{t}^{k}\right|>\varepsilon_{n}\right)$.

Let $P_{n}(x)$ be the upper bound of $\frac{1}{j}$ where $j$ is a positive integer satisfying 
$\sup _{k, h>j^{-1}} e_{n}(x, h, k)<\varepsilon_{n}$. It is clear that $P_{n}(x)$ is $\mathfrak{F}_{S}$-measurable and $P_{n}(x) \rightarrow 0$ as $n \rightarrow \infty$; so that $A_{t}^{P_{n}\left(x_{0}(\omega)\right)}$ is of $\mathfrak{A}^{+}$and satisfies

$$
\sum_{n=1}^{\infty} P_{x}\left(\sup _{t}\left|A_{t}^{P_{n}\left(x_{0}\right)}-A_{t}^{P_{n+1}\left(x_{0}\right)}\right|>\varepsilon_{n}\right)<+\infty
$$

Set $\widetilde{\Omega}=\left\{\omega ; A_{t}^{P_{n}\left(x_{0}(\omega)\right)}(\omega)\right.$ converges uniformly as $n$ tends to infinity $\}$. Then by Borel-Cantelli's lemma, $\widetilde{\Omega}$ is $\mathfrak{F}$-measurable set and $P_{x}(\widetilde{\Omega})=1$ holds for every $x$. Define $A_{t}(\omega)$ as $\lim _{n \rightarrow \infty} A_{t}^{P_{n}\left(x_{0}(\omega)\right)}(\omega)$ for $\omega \in \widetilde{\Omega}$, then this $A_{t}$ is the desired one.

The uniqueness of the above $A_{t}$ immediately follows from

Lemma 8.2. Let $A_{t}$ and $B_{t}$ be of $\mathfrak{A}_{U}^{+}$generating the same supermartingale. Then $A_{t}=B_{t}$.

Proof. Let $Y_{t}(\omega)$ be a bounded process adapted to $\left\{\mathfrak{F}_{t}\right\}$ and continuous from the left. Set $C_{t}=A_{t}-B_{t}$. Let $\left\{T_{n}\right\}, T_{n} \geq S$ be an $\left(\varepsilon, Y_{t}\right)$-chain. Then, since $\left(C_{t}, \mathfrak{F}_{t}\right)$ is a martingale,

$$
E_{x}\left(Y_{T_{n}}\left(C_{T_{n+1}}-C_{T_{n}}\right) / \mathfrak{F}_{S}\right)=0 \text { a.s. } P_{x} .
$$

Letting $\varepsilon \rightarrow 0$, we obtain

$$
E_{x}\left(\int_{(s, t]} Y_{u} d C / \mathfrak{F}_{s}\right)=0 \text { a.e. } P_{x}
$$

The above formula also holds when $Y_{u}$ is of the form $g\left(x_{u}\right), g \in C(S)$ because $C$ has no common jumps with sample path $x_{t}$. Therefore, we get

$$
E \cdot\left(\int_{s}^{\infty} e^{-\alpha u} G_{\alpha} f\left(x_{u}\right) d C_{u} / \mathfrak{F}_{s}\right)=0 .
$$

But the left-hand of the above is

$$
\begin{gathered}
E \cdot\left(\int_{(s, \infty)} d C_{u} E \cdot\left(\int_{0}^{\infty} e^{-\alpha(t+u)} f\left(x_{t+u}\right) d t / \mathfrak{F}_{u}\right) / \mathfrak{F}_{s}\right) \\
=E \cdot\left(\int_{(s, \infty)} d C_{u} \int_{0}^{\infty} e^{-\alpha(t+u)} f\left(x_{t+u}\right) d t / \mathfrak{F}_{s}\right) \\
=E \cdot\left(\int_{s}^{\infty} e^{-\alpha t} f\left(x_{t}\right) d t\left(C_{t}-C_{s}\right) / \mathfrak{F}_{s}\right),
\end{gathered}
$$

so that we have

$$
E\left(f\left(x_{t}\right)\left(C_{t}-C_{s}\right) / \mathfrak{F}_{s}\right)=0
$$


Now by a similar argument as the proof of Theorem 4.2 we get $C_{t} \equiv 0$.

Now we will give the

Proof of Theorem 8. 2. The "if" part of (2) is clear from the quasi-left continuity of sample paths. Suppose that $T$ is accessible. It is known [9] that, for each fixed $x, T$ is left approximable i.e., there exists an increasing sequence of stopping times $\left\{T_{n}^{x}\right\}$ such that $T_{n}^{x}<T$ and $T=\lim _{n \rightarrow \infty} T_{n}^{x}$ hold a.e. $P_{x}$. The quasi-left continuity of sample paths implies (8. 3) immediately.

We shall prove the "only if" part of (2). Define

$$
U_{t}=\chi_{\{t \geqq T>0\}}, \quad U_{t}^{1}=\chi_{\left\{t \geqq T>0 \text { and } x_{T}=x_{T-}\right\}} \text { and } U_{t}^{2}=U_{t}-U_{t}^{1} .
$$

Since the supermartingale $X_{t}$ generated by $U_{t}$ is regular, the supermartingales $X_{t}^{i}(i=1,2)$ are also regular. Thus there exists $V_{t}$ of $\mathfrak{A}$ generating $X_{t}^{1}$, so that $V_{t}=U_{t}^{1}$ by Lemma 1.2. Therefore $U_{t}^{1}=0$ i.e., $U_{t}=U_{t}^{2}$, which means (8. 4). The "if" part of (1) can be obtained from "only if" part of (2). Now Let $T$ be any stopping time. We define $T_{1}$ by $T$ if $x_{T}=x_{T-}$ and by $+\infty$ if $x_{T} \neq x_{T-}$. In the same way, we define $T_{2}$ by $T$ if $x_{T} \neq x_{T-}$ and by $+\infty$ if $x_{T}=x_{T-}$. Then $T_{1}$ is accessible, $T_{2}$ totally inaccessible and satisfies $T=\min \left(T_{1}, T_{2}\right)$. Thus we have accomplished the proof.

Finally we will give the

Proof of Theorem 8. 1. When $X_{t}$ is regular, we have already proved the theorem in Lemma 8. 1. Suppose that $X_{t}$ is not necessarily regular. For $\varepsilon>0$, we define a sequence of stopping times inductively as

$$
T_{0}^{\varepsilon}=0, T_{n}^{\varepsilon}=\inf \left\{t \geqq T_{n-1}^{\varepsilon} ;\left|X_{t}-X_{t-}\right|>\varepsilon\right\} n \geqq 1
$$

and $T_{n}^{\prime \varepsilon}$ as the accessible part of $T_{n}^{\varepsilon}$. Set

$$
A_{t}^{\varepsilon}=\sum_{T_{n}^{\varepsilon} \leqq t}\left(X_{T_{n}^{\prime} \varepsilon}-X_{T_{n-}^{\prime \varepsilon}}\right)
$$

and $A_{t}^{j}=\lim _{\varepsilon \downarrow 0} A_{t}^{\varepsilon}$. It is clear that $A_{t}^{j}$ is of $\mathfrak{U}_{U}^{+}$. Set $\left.X_{t}^{j}=E\left(A_{\infty}^{j} / \mathfrak{F}_{t}\right)-A_{t}^{j}\right\}$. Then we can show that $X_{t}^{c}=X_{t}-X_{t}^{j}$ is regular supermartingale, so that there exists a unique $A_{t}^{c}$ of $\mathfrak{U}_{c}^{+}$generating $X_{t}$. Thus $A_{t}=A_{t}^{c}+A_{t}^{j}$ is what we want.

Remark. If, in Th. 8. 1, $X_{t}$ is an a.f. then $A_{t}$ is an a.f. as is easily seen from the above construction of $A_{t}$. 


\section{REFERENCES}

[1] P. Courrège, Intégrales stochastiques et martingales de carré intégrable, Seminaire Brelot-Choquet-Deny 7e annee (1962/63) 7.

[2 ] J.L. Doob, Stochastic processes, New York (1953).

[3] L.E. Dubins, G. Schwarz, On continuous martingales, Proc. Nat. Acad. Sci. U.S.A. 53(1965), 913-916.

[4] K. Itô, Lectures on stochastic processes, Tata Institute of Fundamental Research, Bombay (1961).

[5] K. Itô, S. Watanabe, Transformation of Markov process by multiplicative functional, Ann. Inst. Fourier, Grenoble 15(1965), 13-30.

[6] H. Kunita, T Watanabe, Notes on transformations of Markov processes connected with multiplicative functionals, Mem. Fac. Sci. Kyushu Univ. 17(1963), 181-191.

[ 7 ] P. Lévy, Theorie de l'addition des variables aleatoires, Paris (1937).

[8] P.A. Meyer, A decomposition theorem for supermartingales, Illinois J. Math. 6(1962), 193-205.

[9] P.A. Meyer, Decompositions of supermartingales; The uniqueness theorem, Illinois J. Math. 7(1963), 1-17.

[10] M. Motoo, S. Watanabe, On a class of additive functionals of Markov process, J. Math. Kyoto Univ. 4(1965), 429-469.

[11] S. Watanabe, On discontinuous additive functionals and Lévy measures of a Markov process, Japanese J. Math. 36(1964), 53-70.

[12] A.D. Wentzel, Additive functionals of multidimensional Wiener process, D.A.N. SSSR, 139(1961), 13-16.

University of Illinois, Nagoya University

and

Stanford University, Kyoto University 